Article

\title{
Formyl Peptide Receptor 1 Signaling in Acute Inflammation and Neural Differentiation Induced by Traumatic Brain Injury
}

\author{
Roberta Fusco $^{1,+}+\mathbb{D}$, Enrico Gugliandolo ${ }^{1,+} \mathbb{D}$, Rosalba Siracusa ${ }^{1,+}\left(\mathbb{D}\right.$, Maria Scuto ${ }^{2}(\mathbb{D}$, \\ Marika Cordaro ${ }^{1}$ (D), Ramona D'Amico ${ }^{1}{ }^{(D}$, Maurizio Evangelista ${ }^{3}$, Angelo Peli ${ }^{4}$, \\ Alessio Filippo Peritore ${ }^{1}$ (D), Daniela Impellizzeri ${ }^{1}$ (D), Rosalia Crupi ${ }^{1}$ (D), \\ Salvatore Cuzzocrea ${ }^{1,5, * \mathbb{D}}$ and Rosanna Di Paola ${ }^{1, * \mathbb{D}}$
}

1 Department of Chemical, Biological, Pharmaceutical and Environmental Sciences, University of Messina, Viale Ferdinando Stagno D'Alcontres, n 31, 98166 Messina, Italy; rfusco@unime.it (R.F.); egugliandolo@unime.it (E.G.); rsiracusa@unime.it (R.S.); cordarom@unime.it (M.C.); rdamico@unime.it (R.D.); aperitore@unime.it (A.F.P.); dimpellizzeri@unime.it (D.I.); rcrupi@unime.it (R.C.)

2 Department of Biomedical and Biotechnological Sciences, University of Catania, Piazza Università, n 2, 95131 Catania, Italy; mary-amir@hotmail.it

3 Institute of Anaesthesiology and Reanimation, Catholic University of the Sacred Heart, A Gemelli Hospital, 00168 Roma, Italy; maurizio.evangelista@unicatt.it

4 Department of Veterinary Medical Sciences, Alma Mater Studiorum, University of Bologna, Via Zamboni n 33, 40126 Bologna, Italy; angelo.peli@unibo.it

5 Department of Pharmacological and Physiological Science, Saint Louis University School of Medicine, 1402 South Grand Blvd, St. Louis, MO 63104, USA

* Correspondence: salvator@unime.it (S.C.); dipaolar@unime.it (R.D.P.); Tel.: +39-90-6765-208 (S.C. \& R.D.P.)

+ These authors contributed equally to this work.

Received: 23 July 2020; Accepted: 18 August 2020; Published: 20 August 2020

check for updates

\begin{abstract}
Traumatic brain injury (TBI) is a shocking disease frequently followed by behavioral disabilities, including risk of cerebral atrophy and dementia. N-formylpeptide receptor 1 (FPR1) is expressed in cells and neurons in the central nervous system. It is involved in inflammatory processes and during the differentiation process in the neural stem cells. We investigate the effect of the absence of Fpr1 gene expression in mice subjected to TBI from the early stage of acute inflammation to neurogenesis and systematic behavioral testing four weeks after injury. C57BL/6 animals and Fpr1 KO mice were subjected to TBI and sacrificed $24 \mathrm{~h}$ or four weeks after injury. Twenty-four hours after injury, TBI Fpr1 KO mice showed reduced histological impairment, tissue damage and acute inflammation (MAPK activation, NF- $\mathrm{B}$ signaling induction, NRLP3 inflammasome pathway activation and oxidative stress increase). Conversely, four weeks after TBI, the Fpr1 KO mice showed reduced survival of the proliferated cells in the Dentate Gyrus compared to the WT group. Behavioral analysis confirmed this trend. Moreover, TBI Fpr1 KO animals displayed reduced neural differentiation (evaluated by beta-III tubulin expression) and upregulation of astrocyte differentiation (evaluated by GFAP expression). Collectively, our study reports that, immediately after TBI, Fpr1 increased acute inflammation, while after four weeks, Fpr1 promoted neurogenesis.
\end{abstract}

Keywords: traumatic brain injury; animal model; neurogenesis; inflammation

\section{Introduction}

$\mathrm{N}$-formylpeptide receptor 1 (FPR1) is a member of a small family of 7-transmembrane G protein-coupled receptors (GPCRs), called the FPR family, that is responsible of several host defense 
reactions [1,2]. $\mathrm{N}$-formylpeptide receptor 1 is mainly expressed on sentinel cells with chemotactic or phagocytic activity, like monocytes [3,4], neutrophils [3,5], dendritic cells $[4,6]$ and macrophages $[4,7]$. Moreover, it is expressed also on non-phagocytic and non-mobile sentinel cells like endothelial cells [8,9], epithelial cells [10,11], neurons [12] and glia [13-15]. It binds several ligands, such as the mitochondrial and pro-inflammatory bacterial $\mathrm{N}$-formylpeptides, as well as the anti-inflammatory agonists lipoxin A4 and annexin-1 [2]. Furthermore, ligands for Fpr1 were detected during inflammatory processes and may stimulate several responses, such as phagocytosis, chemotactic migration, degranulation and free oxygen species production [2]. Traumatic brain injury (TBI) is one of the most important public health issues, clinically considered a "silent epidemic" because the derived problems are not immediately visible [16]. Research has greatly clarified the mechanisms underlying the TBI pathology [17]. These involve a primary insult caused by direct biomechanical forces and a secondary insult that results in brain damage and death following TBI [17-19]. TBI can produce physical and behavioral symptoms and result in complete recovery or permanent disability. In addition, secondary events associated with TBI can influence the life quality of patients [20]. Patients may have a decline in cognitive functions [21,22], risk of cerebral atrophy and dementia [23]. Traumatic injury is a widely used animal model that directly relate to the common clinical problem in humans. After controlled cortical impact, the acute inflammatory response induces the activation of nuclear transcriptional factors, assembly of pro-inflammatory complexes, release of inflammation mediators and increase in oxidative stress [24,25]. In animals, TBI also induces secondary processes, leading to neurological disorders such as learning and memory impairment, and seizures [26]. In particular, four weeks after injury, TBI increases neurogenesis [27-29]. Several studies have found that FPR1 was expressed in neurons [12,30,31]. In particular, it has been described that the FPR1 expression was induced during neuronal differentiation. FPR1 activation triggers the activation NFkB and STAT3 transcriptional factors and signaling molecules, such MAPK, PLC and PLD. Beyond the inflammatory functions, evidence for different jobs has been lacking for Fpr1 [32]. In this regard, we decided to investigate the effect of the absence of Fpr1 gene expression in mice subjected to traumatic brain injury from an early stage of acute inflammation to neurogenesis and systematic behavioral testing four weeks after injury.

\section{Materials and Methods}

\subsection{Animals}

Adult formyl peptide receptor 1 gene-deficient mice on the C57BL/6 genetic background and C57BL/6 animals were used as the wild-type controls [33-35]. Male, 10-week-old mice of the strains Fpr1-/- and wild-type C57/BL6 (from William Harvey Research Institute, Barts and The London School of Medicine, London, UK, purchased from Envigo, Milan, Italy) were used in this study. The University of Messina Review Board for animals' care approved the study. All in vivo experiments followed the new regulations of the USA (Animal Welfare Assurance No A5594-01), Europe (EU Directive 2010/63), Italy (D.Lgs 2014/26) and the ARRIVE guidelines.

\subsection{Induction of Experimental Traumatic Brain Injury TBI}

Mice were anesthetized with an intraperitoneal injection (i.p.) of ketamine and xylazine (2.6 and $0.16 \mathrm{mg} / \mathrm{kg}$ body weight, respectively). Traumatic brain injury was performed by a controlled cortical impactor (CCI) as already described [36]. Briefly, a craniotomy was made encompassing bregma and lambda and between the sagittal suture and the coronal ridge of the right hemisphere, using a Micro motor hand piece and drill. The ensuing bone flap was removed and on the exposed cortex a cortical contusion was produced using the controlled impactor device Impact OneTM Stereotaxic impactor a for CCI (Leica, Milan, Italy) [37]. Subsequently, the skin incision was sutured, and 2\% lidocaine jelly was applied to the lesion to minimize any possible discomfort. Both the WT and FPR1-deficient animals were treated with penicillin $(40,000 \mathrm{U} / \mathrm{kg})$ for five days after TBI to rule out confounding effects by bacterial infections (in the long-term series) [38]. 


\subsection{Experimental Groups}

The mice were randomly divided into four groups $(n=25)$ :

1. TBI WT group: mice were subjected to CCI as described above.

2. TBI Fpr1 KO group: Fpr1 KO mice were subjected to CCI as well as the WT group.

3. Sham WT group: Mice were subjected to the surgical procedures as per the above group (anesthesia and craniotomy) except that the impact tip was not applied.

4. Sham Fpr1 KO group: Mice were subjected to the surgical procedures as per the above group (anesthesia and craniotomy) except that the impact tip was not applied.

In order to analyze the effect of the Fpr1 gene deletion of animals subjected to traumatic brain injury, two experiments were carried out $(n=10)$ :

Exp 1-to investigate the early stage of acute inflammation, animals were sacrificed at $24 \mathrm{~h}$ after TBI. Exp 2-to investigate the neurogenesis, animals were sacrificed four weeks after the injury.

\subsection{Open Field}

Twenty-four days after the traumatic brain injury, the Open Field Test was used for evaluating locomotor activity [39]. The apparatus consisted of a Plexiglas box $50 \mathrm{~cm} \times 50 \mathrm{~cm}$ with its floor separated into 16 squares. The center was defined by four squares and the squares along the wall defined the periphery. During the test, the mouse was located in the center of the box, and the movement of the mouse was observed for $5 \mathrm{~min}$. The movement was scored as a line crossing when a mouse removed all paws from one square and entered another. The number of crossings and the time spent in the center were calculated and scored.

\subsection{Social Interaction Test}

The social interaction test was performed twenty-three days after the traumatic brain injury using a three-chambered apparatus (polycarbonate $80 \mathrm{~cm} \times 31.5 \mathrm{~cm}$ ) divided into three compartments. It consisted of three trials of ten minutes. Initially, a mouse was acclimated in an empty arena for $5 \mathrm{~min}$. In the second phase, the experimental mouse was exposed to an object, one of the empty wired cages and a wired cage covering a stimulus mouse. Time spent engaging in investigatory behavior with the novel mouse and the frequency of the investigatory behavior with the novel mouse was recorded. All testing happened during the dark phase (21:00-03:00 h) under illumination with red light.

\subsection{Novel Object Recognition Test}

The experiment was conducted as previously described [40] and performed in a black empty box in a quiet environment. The mouse was replaced in the box, and its behavior was observed for $10 \mathrm{~min}$. The total time the mouse spent exploring each object was recorded. The exploration time included the distance between the object and the nose tip when the mouse sniffed the object from less than $2 \mathrm{~cm}$, and the times the front paw or nose directly touched the object. Walking near the object was not considered exploratory behavior. A solution of $90 \%$ ethanol was used to eliminate odors between different animals (to avoid olfactory cues from affecting the exploratory behavior of other animals).

\subsection{Morris Water Maze Test}

The water maze test was conducted as previously described [41] after twenty-four days from TBI. The device was a stainless-steel sink, with a height of $50 \mathrm{~cm}$ and diameter of $100 \mathrm{~cm}$, containing four quadrants. A circular platform with a height of $27 \mathrm{~cm}$ and a diameter of $9 \mathrm{~cm}$ was in the center of the platform quadrant, and the position did not change throughout the experiment. Milk was added to make the water opaque and the temperature was kept at $23^{\circ} \mathrm{C}$. On the first day, a visual platform experiment was performed. During the following 2-5 days, the navigation experiment was performed. 
One day after the navigation experiment, the platform was removed for the test. The mouse was located in the water in the same quadrant. The time spent in the target quadrant and the number of entries into it were recorded. All experiments were carried out between 9.00 a.m. and 5.00 p.m.

\subsection{Histological Examination}

Brain tissues were fixed in formalin solution, dehydrated by graded ethanol and embedded in paraffin. Sections of $5-\mu \mathrm{m}$ thickness were collected on the glass slides, deparaffinized, and then stained with hematoxylin and eosin (H\&E) [42,43]. Histopathologic changes of the gray matter were evaluated on a six-point scale [44].

\subsection{Assessment of Lesion Volume}

At $24 \mathrm{~h}$ and four weeks after CCI, animals were euthanized, and the brain tissues were frozen and sectioned in coronal sections $(300 \mu \mathrm{m})$. Samples were stained, and the area of injured hemisphere was scored using image analysis software. The hemispheric volume was evaluated by summing the area of each section and multiplying it by 0.5 . Lesion volume $\left(\mathrm{mm}^{3}\right)$ was showed as the difference between the injured and uninjured hemisphere volume. For the analysis, $n=5$ animals from each group were employed.

\subsection{Myeloperoxidase Activity}

Myeloperoxidase (MPO) activity, an indicator of neutrophils accumulation, was determined as previously published [45,46]. MPO activity was expressed in U per gram weight of wet tissue and was defined as the quantity of enzyme degrading $1 \mu \mathrm{mol}$ of peroxide $\min ^{-1}$ at $37^{\circ} \mathrm{C}$. For the analysis, $n=5$ animals from each group were employed.

\subsection{Western Blot Analysis}

Western blot analysis was executed on tissues harvested $24 \mathrm{~h}$ and four weeks after TBI [46]. Cytosolic and nuclear extracts were divided as described previously [47,48]. Membranes were probed with specific Abs: anti-NF-kB p-65 (1:1000; Santa Cruz Biotechnology, Heidelberg, Germany) or with IkB- $\alpha$ (1:1000; Santa Cruz Biotechnology), or with anti-NLRP3(1:500; Santa Cruz Biotechnology), or with anti-ASC (1:500; Santa Cruz Biotechnology), or with anti-Caspase-1 (1:500; Santa Cruz Biotechnology), or with iNOS (1:500; Transduction Laboratories, Milan, Italy), or with anti-MnSod (1:500 Millipore, Milan, Italy), or with anti p-AKT (1:500; Santa Cruz Biotechnology), or with anti AKT (1:500; Santa Cruz Biotechnology), or with anti $\beta$-III tubulin (1:500; Santa Cruz Biotechnology), or with anti GFAP (1:500; Santa Cruz Biotechnology) or with anti-pp38 (1:500; Santa Cruz Biotechnology) or with anti-p38 (1:500; Santa Cruz Biotechnology) or with anti-pERK (1:500; Santa Cruz Biotechnology) or anti-ERK (1:500; Santa Cruz Biotechnology) or with anti-Cox-2 (1:500; Santa Cruz Biotechnology) or anti-PGE synthase (1:500; Santa Cruz Biotecnology) or anti-PGD2 synthase (1:500; Santa Cruz Biotecnology) in 1xPBS, $5 \% \mathrm{w} / \mathrm{v}$ nonfat dried milk, $0.1 \%$ Tween- 20 at $4{ }^{\circ} \mathrm{C}$, overnight. To control that, the blots were loaded with equal amounts of proteins and also were probed with antibodies against b-actin protein (cytosolic fraction 1:500; Santa Cruz Biotechnology) or lamin A/C (nuclear fraction 1:500 Sigma-Aldrich Corp., Milan, Italy). Signals were examined with an enhanced chemiluminescence (ECL) detection system reagent according to the manufacturer's instructions (Thermo, Monza, Italy). The relative expression of the protein bands was quantified by densitometry with BIORAD ChemiDocTM XRS + software and standardized to the b-actin and lamin A/C levels. (All original western blots can be available in Figure S1). 


\subsection{Bromodeoxyuridine (BrdU) Treatment}

To assess newly-generated neurons and proliferated cells in Dentate Gyrus (DG), mice received $\mathrm{BrdU}(50 \mathrm{mg} / \mathrm{kg}$, i.p. dissolved in saline) every day for seven days after TBI [49]. BrdU incorporation into cell nuclei was assessed by immunohistochemistry.

\subsection{Immunohistochemical Analysis}

Immunohistochemical analysis was performed as already described [50]. Subsequently, the sections were incubated overnight with an anti-Bromodeoxyuridine (BrdU) antibody (1:100; Santa Cruz Biotechnology) or anti-MPO antibody (1:250; Santa Cruz Biotechnology) or anti-NRLP3 antibody (1:250; Santa Cruz Biotechnology) or anti-COX-2 antibody (1:250; Santa Cruz Biotechnology) or anti-Iba-1 antibody (1:250; Santa Cruz Biotechnology) or anti-GFAP antibody (1:450; Santa Cruz Biotechnology). Sections were washed with PBS and incubated with peroxidase-conjugated bovine anti-mouse IgG, secondary antibody (1:2000 Jackson Immuno Research, West Grove, PA, USA). Specific labeling was provided with a biotin-conjugated goat anti-mouse IgG and avidin-biotin peroxidase complex (Vector Laboratories, Burlingame, CA, USA). Images were collected using a Leica DM6 microscope associated with Leica LAS X Navigator software. The number of positive cells was counted in three sections per animal and presented as the number of positive cells per high-power field.

\subsection{ELISA Analysis of IL-1 $\beta$ and IL-18}

Brains were collected and rinsed with PBS to remove excess blood, chopped into 1-2 mm pieces and homogenized with a tissue homogenizer. A total of $1.0 \mathrm{~mL}$ of Lysis Buffer (R\&D Systems) was added. Brains were lysed at room temperature for $30 \mathrm{~min}$ with gentle agitation and centrifuged to remove debris. An aliquot of each tissue lysate was removed and assayed for levels of IL-1 $\beta$ and IL 18. The levels of IL-1 $\beta$ and IL 18 in tissues surrounding the cortical contusion site were performed by the specific ELISA kits according to the manufacturer's instructions (R\&D Systems, Inc., Minneapolis, MN, USA).

\subsection{Materials}

All compounds used in this study, except where differently specified, were purchased from Sigma-Aldrich Company Ltd.

\subsection{Statistical Evaluation}

All values in the figures and text are expressed as the mean \pm standard error of the mean (SEM) of $N=5$ number of animals. Results were analyzed by two-way ANOVA followed by a Bonferroni post-hoc test for multiple comparisons. Non-parametric data were analyzed with the Fisher's exact test. A $p$-value $<0.05$ was considered significant. ${ }^{*} p<0.05$ vs. Sham WT; ${ }^{\circ} p<0.05$ vs. TBI WT.

\section{Results}

\subsection{Effect of Absence of Fpr1 on Severity of Tissue Damage 24 h Following Traumatic Brain Injury}

No significant histological and macroscopic differences were detected in the brain tissue of Sham WT and Fpr1 KO animals (Figure 1A,C,E for histological score and Figure 1F for lesion volume). Twenty-four hours after TBI injury, the histological analysis of the perilesional area showed in the TBI WT group significant tissue damage, ischemic changes, thickened blood vessels, and gliosis in the brain parenchyma (Figure 1B,E for histological score and Figure 1F for lesion volume). In the TBI Fpr1 $\mathrm{KO}$ group, the histological analysis showed a significantly reduced degree of brain injury compared to the TBI WT group (Figure 1D,E for histological score). Moreover, the absence of an Fpr1 receptor led to a reduction in the lesion volume compared to the TBI WT group (Figure 1F). 

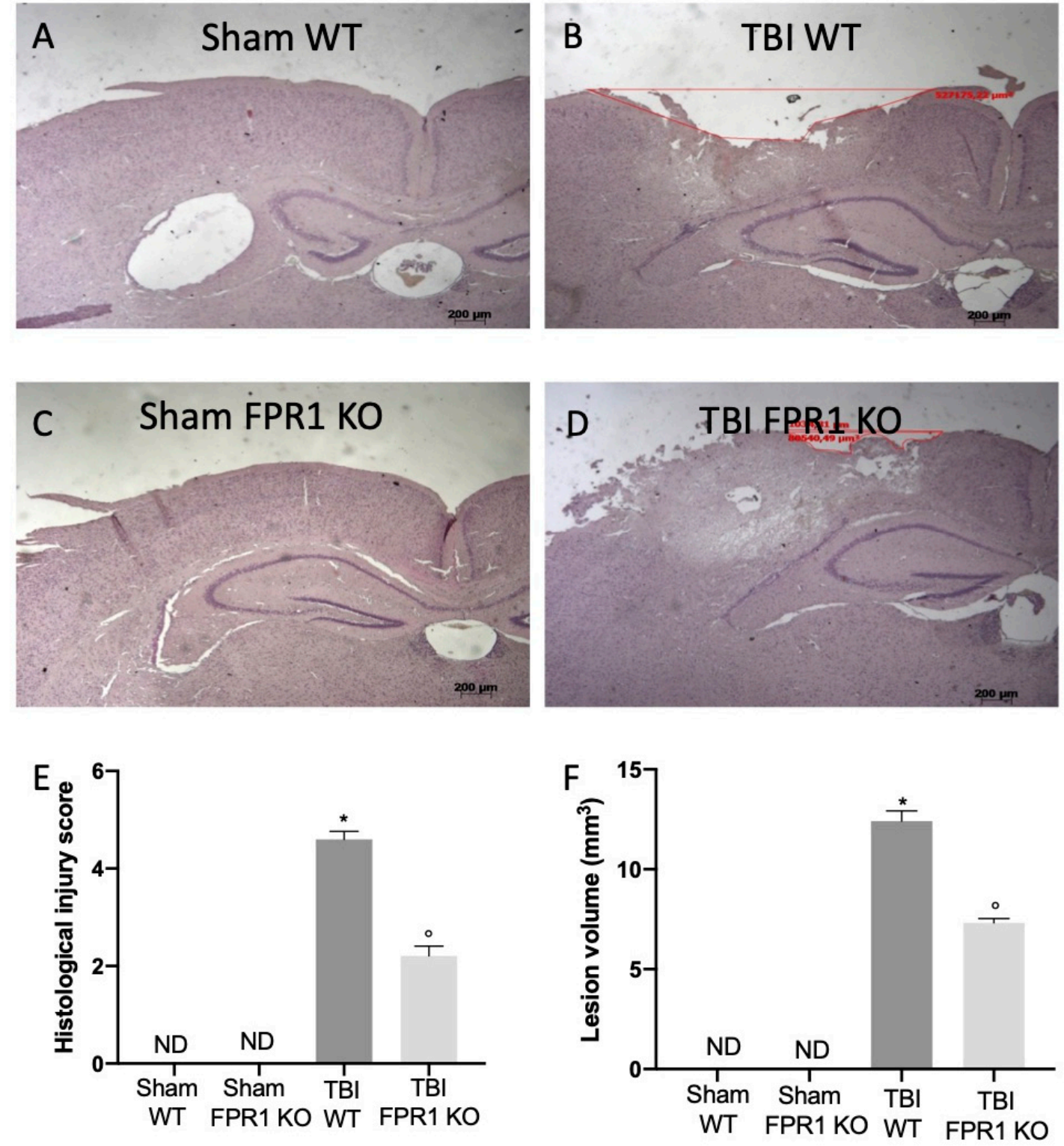

Figure 1. Effect of the absence of Fpr1 on histological alterations: Histological analysis shows no alteration in brain section from mice of the Sham WT group (A) and Sham Fpr1 KO group (C). Traumatic brain injury (TBI) WT group $24 \mathrm{~h}$ after injury displays evident tissue inflammation and disorganization in the perilesional area (B). (D) Fpr1 KO animals subjected to TBI show a significant reduction in the injury compared to the TBI WT group (E). Moreover, the TBI Fpr1 KO group displays a reduced lesion volume compared to TBI WT group as show in (F). For the analyses, $n=5$ animals from each group were employed. Results were analyzed by two-way ANOVA followed by a Bonferroni post-hoc test for multiple comparisons. Non-parametric data were analyzed with Fisher's exact test. ${ }^{*} p<0.05$ vs. Sham WT; ${ }^{\circ} p<0.05$ vs. TBI WT. scale bar $200 \mu \mathrm{m}$.

\subsection{Effect of Absence of Fpr1 on MPO Activity 24 h Following Traumatic Brain Injury}

Traumatic brain injury was characterized by neutrophil infiltration in the tissue, quantified through measurement of MPO activity (Figure 2A). Mice lacking Fpr1 gene expression subjected to TBI showed reduced MPO activity (Figure 2E), compared to the TBI WT group (Figure 2D). No positive staining for MPO was identified in the sham groups (Figure 2B,C). 

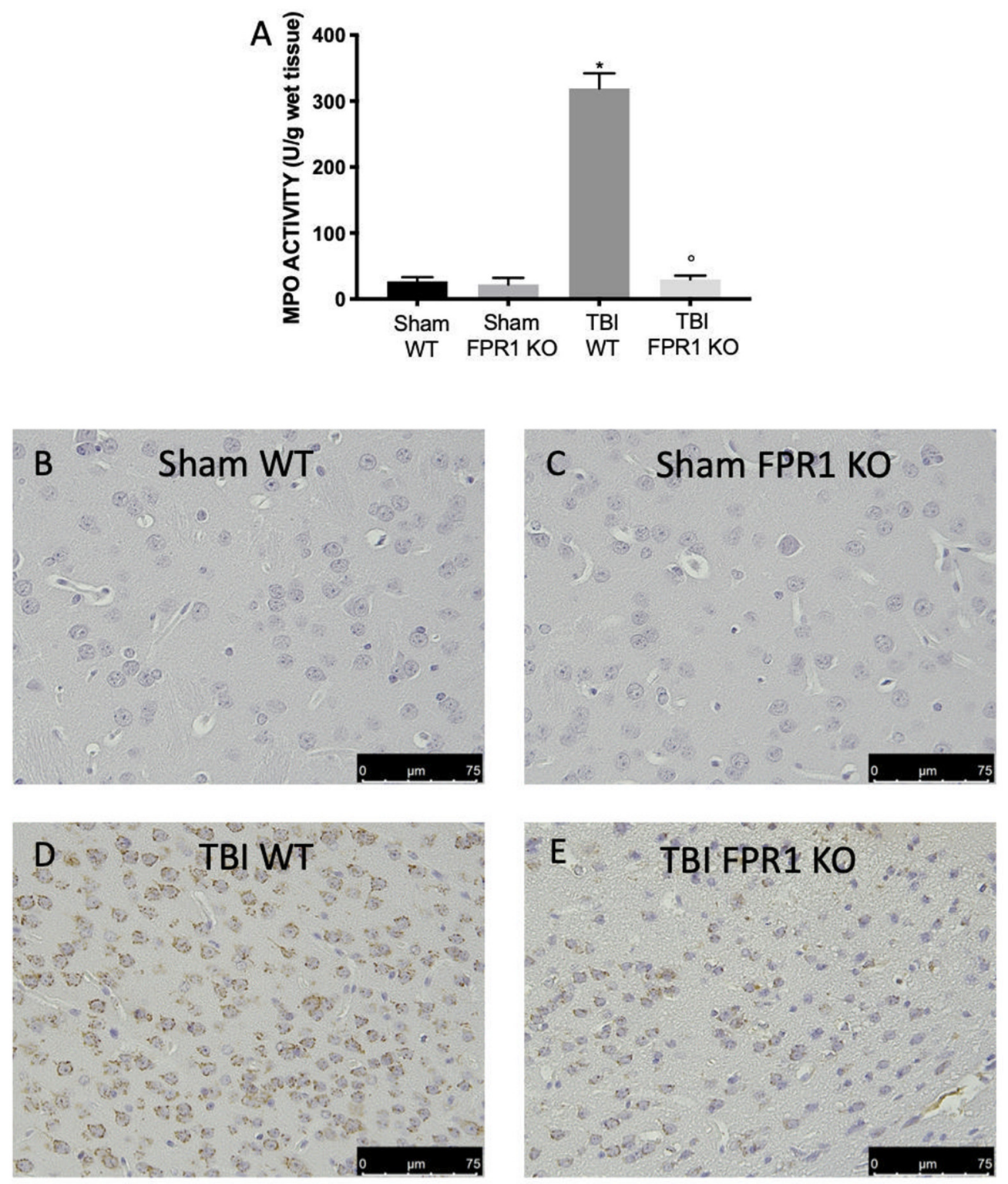

Figure 2. Effect of the absence of Fpr1 on neutrophils accumulation: TBI Fpr1 KO mice showed a reduction in brain MPO activity (A) Immunohistochemical analysis showed an increased number of MPO-positive cells in WT animals subjected to TBI $24 \mathrm{~h}$ after injury (D) compared to the Sham WT group (B) and Sham Fpr1 KO group (C). Fpr1 KO animals $24 \mathrm{~h}$ after TBI shows significantly less MPO-positive cells (E). For the analyses, $n=5$ animals from each group were employed. Results were analyzed by two-way ANOVA followed by a Bonferroni post-hoc test for multiple comparisons. Non-parametric data were analyzed with Fisher's exact test. ${ }^{*} p<0.05$ vs. Sham WT; ${ }^{\circ} p<0.05$ vs. TBI WT. scale bar $75 \mu \mathrm{m}$.

\subsection{Effect of Absence of Fpr1 on MAPK Pathway 24 h Following Traumatic Brain Injury}

P-p38 expression levels, monitored by Western blotting, were considerably increased in tissue collected from TBI WT mice compared to the Sham WT and Fpr1 KO animals. The absence of Fpr1 decreased p-p38 expression in TBI animals (Figure 3A,B). Moreover, traumatic brain injury induced increased ERK phosphorylation in WT animals, while it was remarkably reduced in samples from TBI Fpr1 KO animals $24 \mathrm{~h}$ after injury. (Figure 3C,D). 


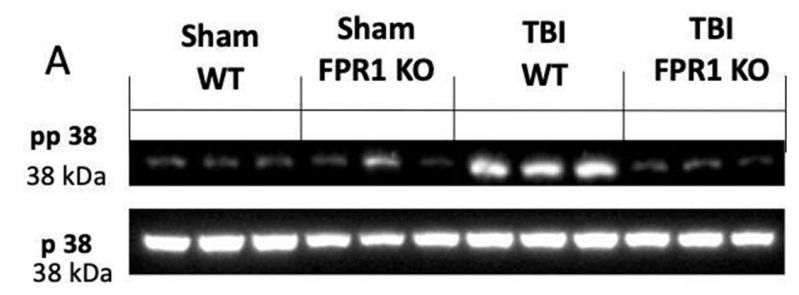

B
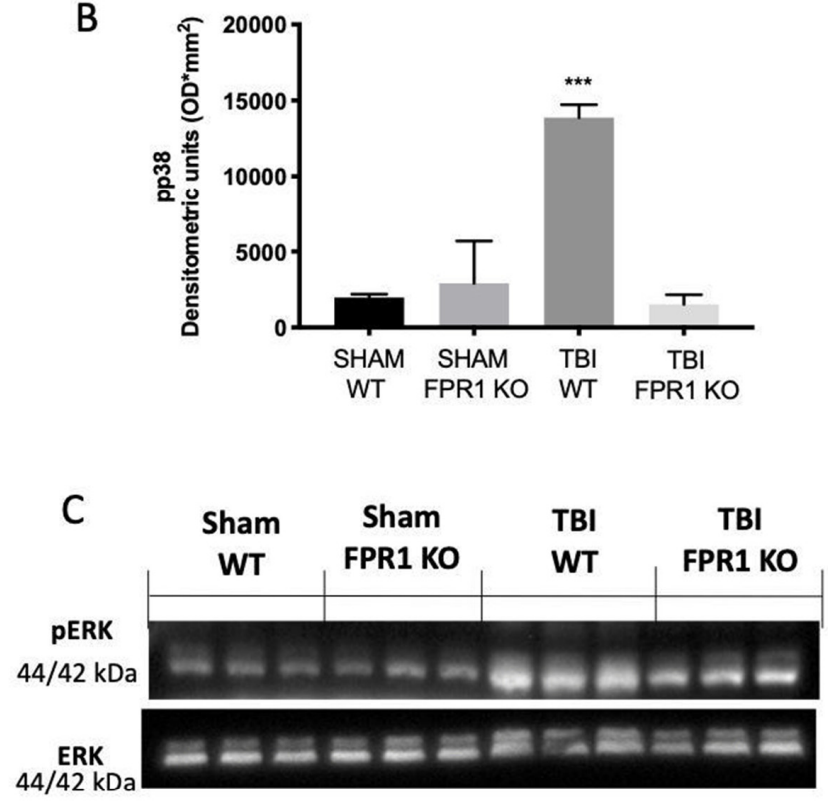

D

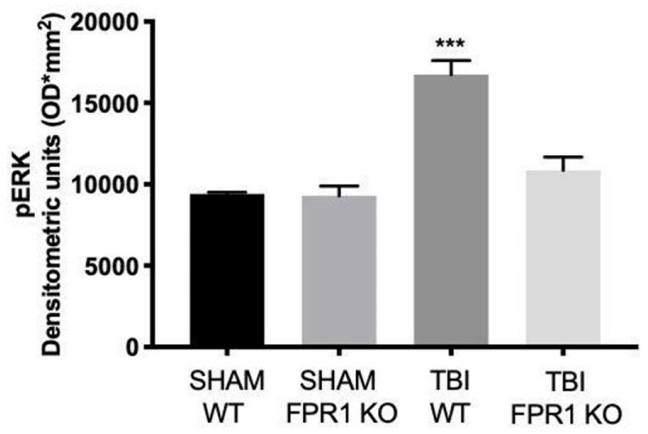

Figure 3. Effect of the absence of Fpr1 on p38 and ERK phosphorylation: Molecular analysis shows an increased p38 (A) and ERK (C) phosphorylation in the TBI WT group $24 \mathrm{~h}$ after injury, compared to the Sham WT group and Sham Fpr1 KO group. TBI Fpr1 KO animals do not show any significant phosphorylation, see densitometric analysis (B,D). For the analyses, $n=5$ animals from each group were employed. Results were analyzed by two-way ANOVA followed by a Bonferroni post-hoc test for multiple comparisons. Non-parametric data were analyzed with Fisher's exact test. ${ }^{* *} p<0.001$ vs. Sham WT.

\subsection{Effect of Absence of Fpr1 on COX-2 and Prostaglandin Expression $24 \mathrm{~h}$ Following Traumatic Brain Injury}

Immunohistochemical analysis showed increased COX-2 expression in TBI WT mice (Figure 4C) compared to the sham WT (Figure 4A) and Fpr1 KO animals (Figure 4B), while TBI Fpr1 KO animals did not show any upregulation (Figure 4D). Western blot analysis also displayed an increased expression of COX-2 (Figure 4E,F) and PGE2 synthase (Figure 4G,H) in brains from TBI WT mice compared to the 
Sham WT and Fpr1 KO animals, while TBI Fpr1 KO animals did not show any increase. PGD2 synthase expression levels decreased in WT animals $24 \mathrm{~h}$ after TBI, compared to the Sham WT and Fpr1 KO animals. In TBI Fpr1 KO animals, PGD2 synthase expression remained at the basal levels (Figure 4I,J).

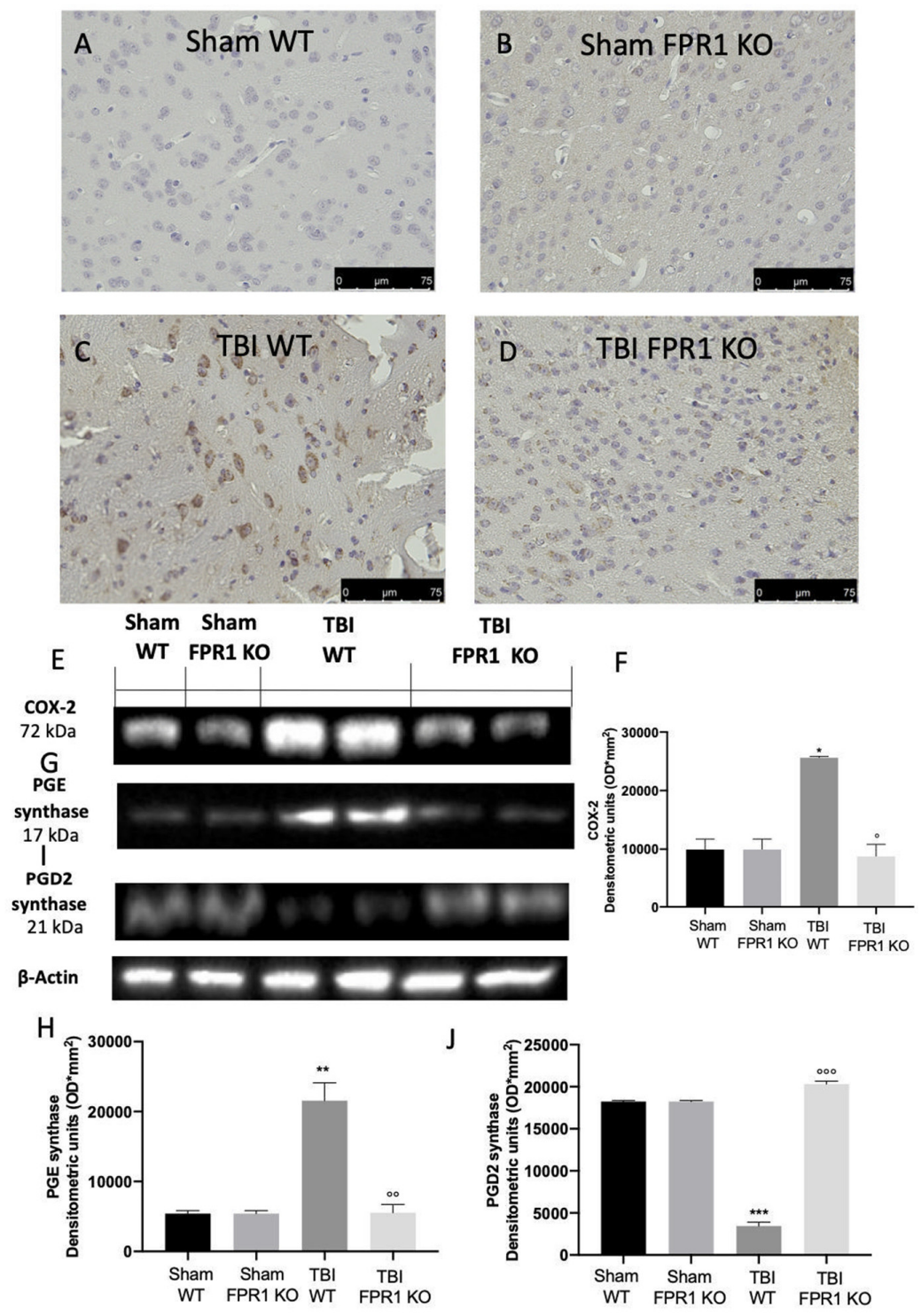

Figure 4. Effect of the absence of Fpr1 on COX-2 and PGE2 and PGD2 synthase: Immunohistochemical analysis displays an increased COX-2 (C) expression in TBI WT mice compared to the sham WT (A) and Fpr1 KO animals (B) while TBI Fpr1 KO animals do not show any increase (D) Western blot analysis also displays an increased COX-2 (E) and PGE2 synthase (G) expression in TBI WT mice compared to the Sham WT and Fpr1 KO animals, while TBI Fpr1 KO animals do not show any increase. PGD2 synthase expression (I) decreases in WT animals $24 \mathrm{~h}$ after TBI, compared to the Sham WT and Fpr1 KO animals. In TBI Fpr1 KO animals, PGD2 synthase expression remain at the basal levels. See densitometric analysis $(\mathbf{F}, \mathbf{H}, \mathbf{J})$ For the analyses, $n=5$ animals from each group were employed. Results were analyzed by two-way ANOVA followed by a Bonferroni post-hoc test for multiple comparisons. Non-parametric data were analyzed with Fisher's exact test. ${ }^{*} p<0.05$ vs. Sham WT; ${ }^{* *} p<0.01$ vs. Sham WT; ${ }^{* * *} p<0.001$ vs. Sham WT; ${ }^{\circ} p<0.05$ vs. TBI WT; ${ }^{\circ \circ} p<0.01$ vs. TBI WT; ${ }^{\circ \circ} p<0.001$ vs. TBI WT. scale bar $75 \mu \mathrm{m}$. 


\subsection{Effect of Absence of Fpr1 on I $\kappa B-\alpha$ and NF- $\kappa B$ Expression $24 h$ after Traumatic Brain Injury}

Nuclear upregulation of NF- $\mathrm{KB}$ is a hallmark of inflammatory brain diseases [51]. In order to investigate the pathway whereby Fpr1 gene deletion could moderate the inflammatory response induced by traumatic brain injury $24 \mathrm{~h}$ afterwards, we checked the IkB- $\alpha$ expression in the cytosol and NF- $\kappa B$ expression into the nucleus (Figure $5 \mathrm{~A}, \mathrm{C}$ ). Western blot analysis showed basal cytosolic expression of IKB- $\alpha$ in brain samples from Sham WT and Fpr1 KO mice, while IKB- $\alpha$ expression was remarkably decreased in samples from TBI WT animals 24 days after injury. In Fpr1 KO animals, a reduction in TBI-induced I $\kappa \mathrm{B}-\alpha$ expression was detected (Figure $5 \mathrm{~A}, \mathrm{~B}$ ). In parallel, NF- $\mathrm{kB}$ expression in the brain nuclear fractions were substantially upregulated $24 \mathrm{~h}$ after TBI, compared to the sham WT and Fpr1 $\mathrm{KO}$ animals, which was reduced in Fpr1 KO mice (Figure 5C,D).

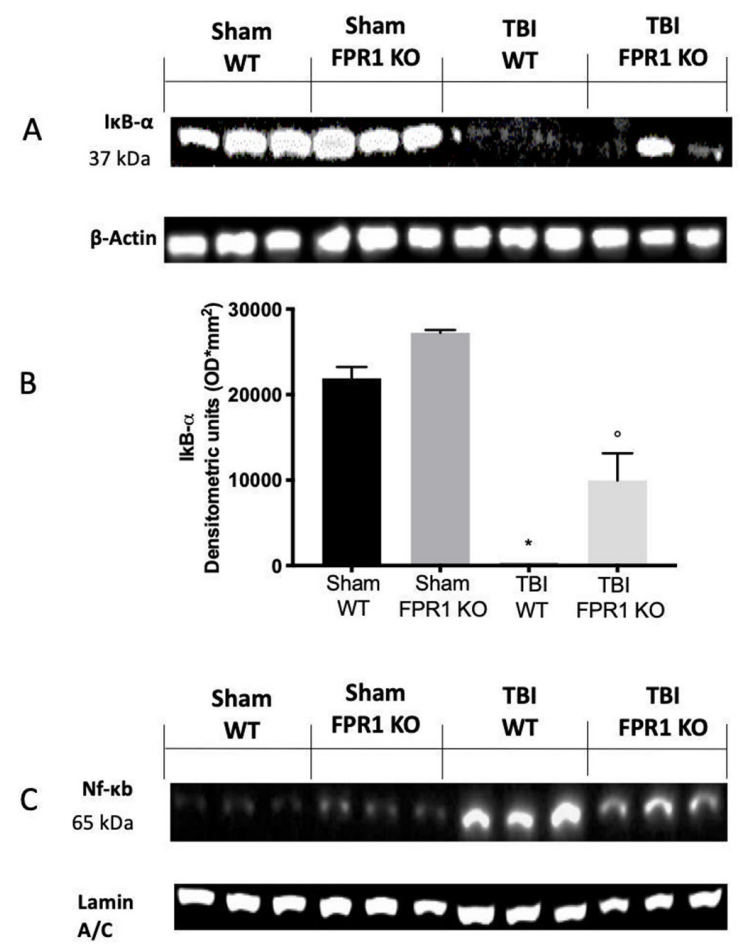

D

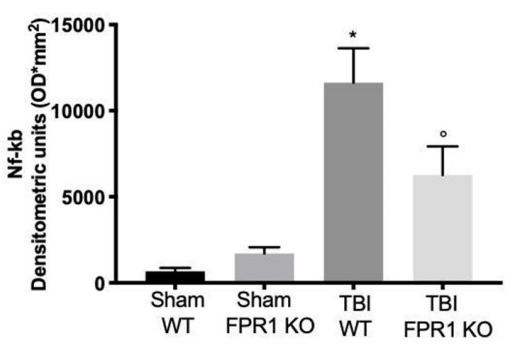

Figure 5. Effect of the absence of Fpr1 on IkB- $\alpha$ and NF-kB p65 expression: Western blot analysis shows basal expression of IkB- $\alpha$ in the Sham WT group and Sham Fpr1 KO group (A). A significant reduction in IkB- $\alpha$ expression in the TBI WT group $24 \mathrm{~h}$ after injury, while TBI Fpr1 KO animals show an increased IkB- $\alpha$ expression; see the densitometric analysis (B). Western blot analysis shows an upregulation of Nf-kb expression in the TBI WT group $24 \mathrm{~h}$ after TBI injury, compared to the Sham WT and Sham Fpr1 KO group (C). Fpr1 KO animals $24 \mathrm{~h}$ after TBI show a reduced Nf-kb translocation into the nucleus compare to the TBI WT group; see the densitometric analysis (D). For the analyses, $n=5$ animals from each group were employed. Results were analyzed by two-way ANOVA followed by a Bonferroni post-hoc test for multiple comparisons. Non-parametric data were analyzed with Fisher's exact test. ${ }^{*} p<0.05$ vs. Sham WT; ${ }^{\circ} p<0.05$ vs. TBI WT. 


\subsection{Effect of Absence of Fpr1 on Inflammasome Components $24 \mathrm{~h}$ after Traumatic Brain Injury}

The activation of the NF- $\mathrm{kB}$ pathway due to the traumatic brain injury led to an increase expression of the inflammasome complex in brain tissue. Immunohistochemical analysis showed increased NLRP3 expression in the TBI WT mice (Figure 6C) compared to the Sham WT (Figure 6A) and Fpr1 KO animals (Figure 6B), while TBI Fpr1 KO animals did not show any upregulation (Figure 6D). In particular, $24 \mathrm{~h}$ after injury, the TBI WT animals showed an upregulation of NLRP3 (Figure 6E,H), ASC (Figure 6F,I) and Caspase-1 (Figure 6G,J) levels compared to the Sham WT and Fpr1 KO animals. Tissues collected from TBI Fpr1 KO mice showed a reduced expression of all the proteins of the inflammasome complex.

\subsection{Effect of Absence of Fpr1 on Oxidative Stress Activation 24 h after Traumatic Brain Injury}

We also evaluated by ELISA analysis the expression of IL-1 $\beta$ and IL-18 activated by the NLRP3 pathway. Both the IL-1 $\beta$ and IL-18 levels were upregulated in TBI Fpr1 KO mice compared to the Sham WT and Fpr1 KO animals (Figure 7A,B). The absence of the Fpr1 receptor also reduced the activation of this interleukins as well as expression of the NLRP3 complex. Next, we evaluated the anti-neuroinflammatory effect of the absence of Fpr1 on oxidative stress activation $24 \mathrm{~h}$ post traumatic brain injury. Twenty-four hours after TBI, the Western blot analysis for iNOS expression displayed an upregulation of its level in WT animals compared to the Sham WT and Fpr1 KO mice, while Fpr1 $\mathrm{KO}$ animals subjected to TBI showed significantly less activation (Figure $7 \mathrm{C}, \mathrm{E}$ ). To test whether Fpr1 modulates the oxidative process, we investigated the brain expression of the anti-oxidant enzyme Mn-SOD. A basal expression of Mn-SOD was found in samples from Sham WT and Fpr1 KO mice. TBI reduced its expression in WT animals while the absence of the Fpr1 receptor significantly restored brain Mn-SOD expression (Figure 7D,F).

\subsection{Effect of Absence of Fpr1 on Astrocytes Activation 24 h after Traumatic Brain Injury}

Astrocytes activation plays a critical role in neuroinflammation. When compared to the Sham WT (Figure 8A,E) and Sham FPR1 KO group (Figure 8B,F), immunohistochemical evaluation of the glial fibrillary acidic protein (GFAP) revealed a significant increase in the TBI WT group in both the cortex and hippocampus, as shown in Figure 8C,G, respectively. In the TBI Fpr1 KO group, there were no significant increase in GFAP-positive cells (Figure 8D,H).

\subsection{Effect of Absence of Fpr1 on Microglia Activation 24 hafter Traumatic Brain Injury}

In order to evaluate the effect of the Fpr1 gene deletion on microglia activation $24 \mathrm{~h}$ after traumatic brain injury, an immunohistochemical analysis was performed. We observed that ionized calcium binding adaptor molecule 1 (Iba1) expression was very low in the Sham WT cortex (Figure 9A) and hippocampus (Figure 9E) and Sham FPR1 KO cortex (Figure 9B) and hippocampus (Figure 9F), while it was increased in the TBI WT cortex (Figure 9C) and hippocampus (Figure 9G). In the TBI Fpr1 KO group there were no significant increase in Iba1-positive cells in both the cortex (Figure 9D) and hippocampus (Figure 9H).

\subsection{Effect of Absence of Fpr1 on Severity of Tissue Damage Four Weeks Following Traumatic Brain Injury}

No significant histological and macroscopic differences were detected in the brain tissue of Sham WT and Fpr1 KO animals (Figure 10A,C,E for histological score and Figure 10F for lesion volume). Four weeks after TBI injury, the histological analysis showed in the TBI WT group significant tissue damage (Figure 10B,E for histological score and Figure 10F for lesion volume). The TBI Fpr1 KO group showed a significantly reduced degree of brain injury compared to the TBI WT group (Figure 10D,E for histological score). Moreover, the absence of the Fpr1 receptor led to a reduction in the lesion volume compared to the TBI WT group (Figure 10F). 

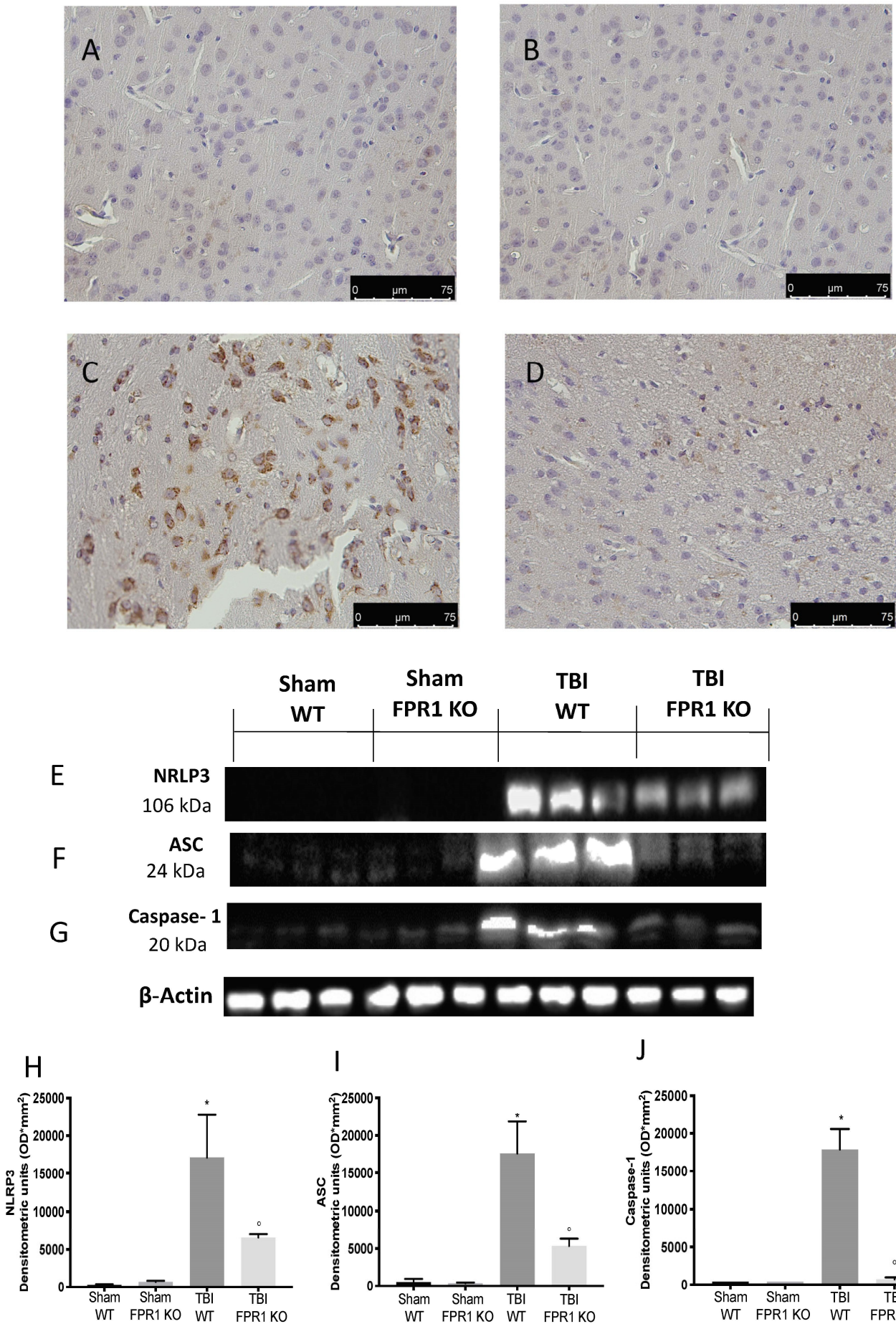

J
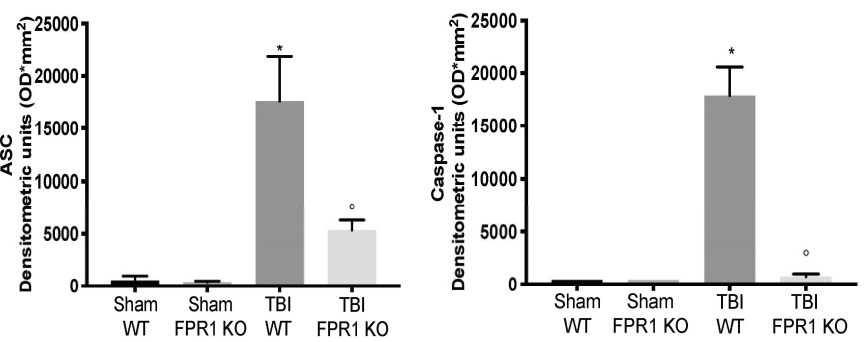

Figure 6. Effect of the absence of Fpr1 on NLRP-3 inflammasome complex activation: Immunohistochemical analysis displays an increased NLRP-3 (C) expression in TBI WT mice compared to the Sham WT (A) and Fpr1 KO animals (B) while TBI Fpr1 KO animals do not show any increase (D) Western blot analysis confirms the upregulation of NLRP3 expression (E) ASC (F) and Caspase-1 (G) in the TBI WT group $24 \mathrm{~h}$ after injury, compared to the Sham WT and Sham Fpr1 KO group. Fpr1 KO animals $24 \mathrm{~h}$ after TBI show reduced levels of NLRP3, ASC and Caspase-1 compare to the TBI WT group; see the densitometric analysis (H-J, respectively). For the analyses, $n=5$ animals from each group were employed. Results were analyzed by two-way ANOVA followed by a Bonferroni post-hoc test for multiple comparisons. Non-parametric data were analyzed with Fisher's exact test. ${ }^{*} p<0.05$ vs. Sham WT; ${ }^{\circ} p<0.05$ vs. TBI WT. scale bar $75 \mu \mathrm{m}$. 

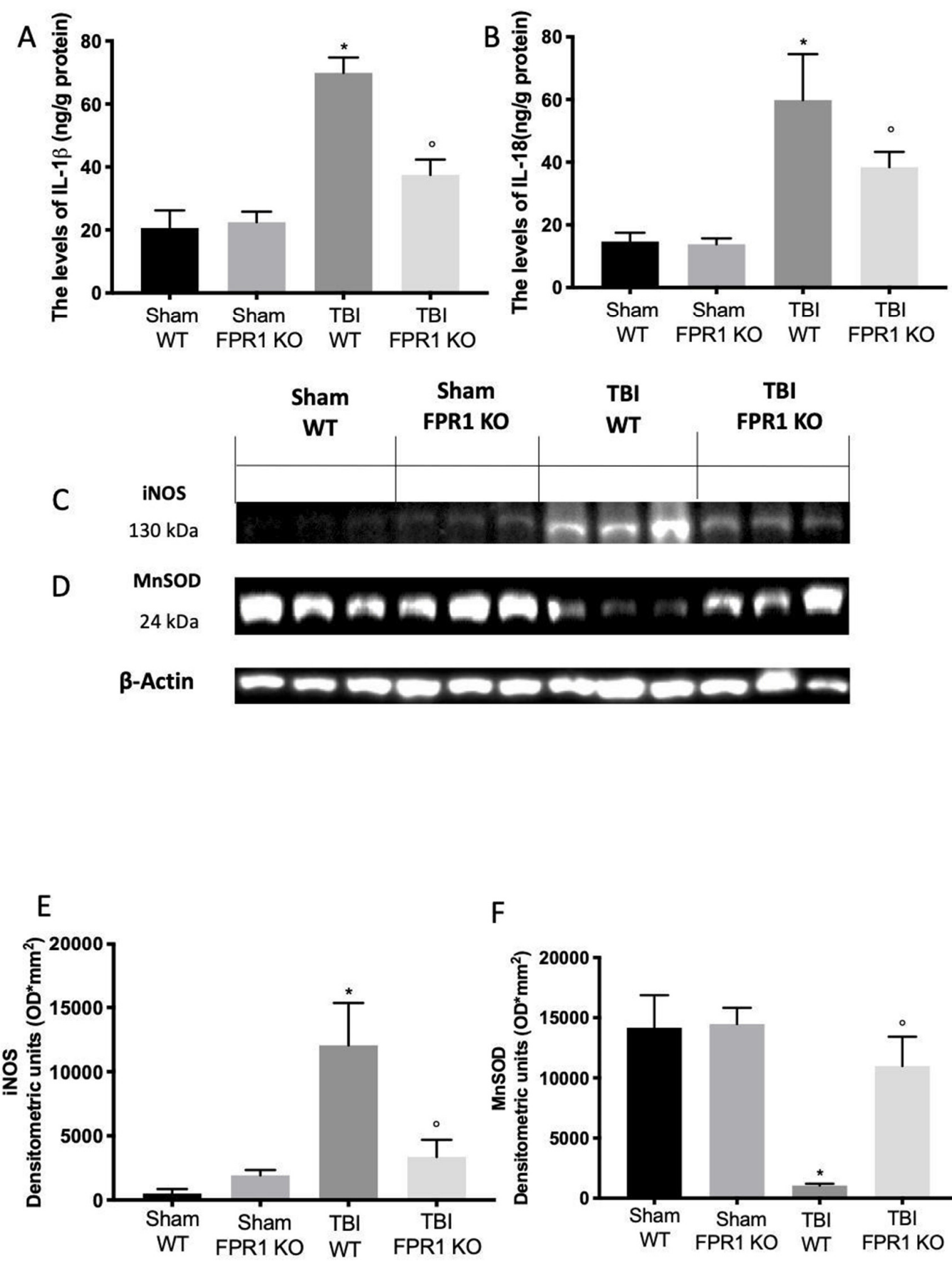

F

Figure 7. Effect of the absence of Fpr1 on IL-1 $\beta$, IL-18 and lipid peroxidation: ELISA analysis shows a positive expression of IL-1 $\beta$ (A) and IL-18 (B) in samples collected from TBI WT animals $24 \mathrm{~h}$ after injury. A substantial reduction of these expressions is detected in samples from TBI Fpr1 KO animals $(\mathbf{A}, \mathbf{B})$ Western blot analysis shows an upregulation of iNOS expression (C) in the TBI WT group $24 \mathrm{~h}$ after injury, compared to the Sham WT and Sham Fpr1 KO group. Fpr1 KO animals $24 \mathrm{~h}$ after TBI show reduced levels of iNOS compare to the TBI WT group; see the densitometric analysis (E) The same analysis shows basal expression of MnSOD (D) in the Sham WT group and Sham Fpr1 KO group. A significant reduction in MnSOD expression is expressed in the TBI WT group $24 \mathrm{~h}$ after injury, while TBI Fpr1 KO animals show a restored expression activation of the anti-oxidant enzyme; see the densitometric analysis (F) For the analyses, $n=5$ animals from each group were employed. Results were analyzed by two-way ANOVA followed by a Bonferroni post-hoc test for multiple comparisons. Non-parametric data were analyzed with Fisher's exact test. ${ }^{*} p<0.05$ vs. Sham WT; ${ }^{\circ} p<0.05$ vs. TBI WT. 

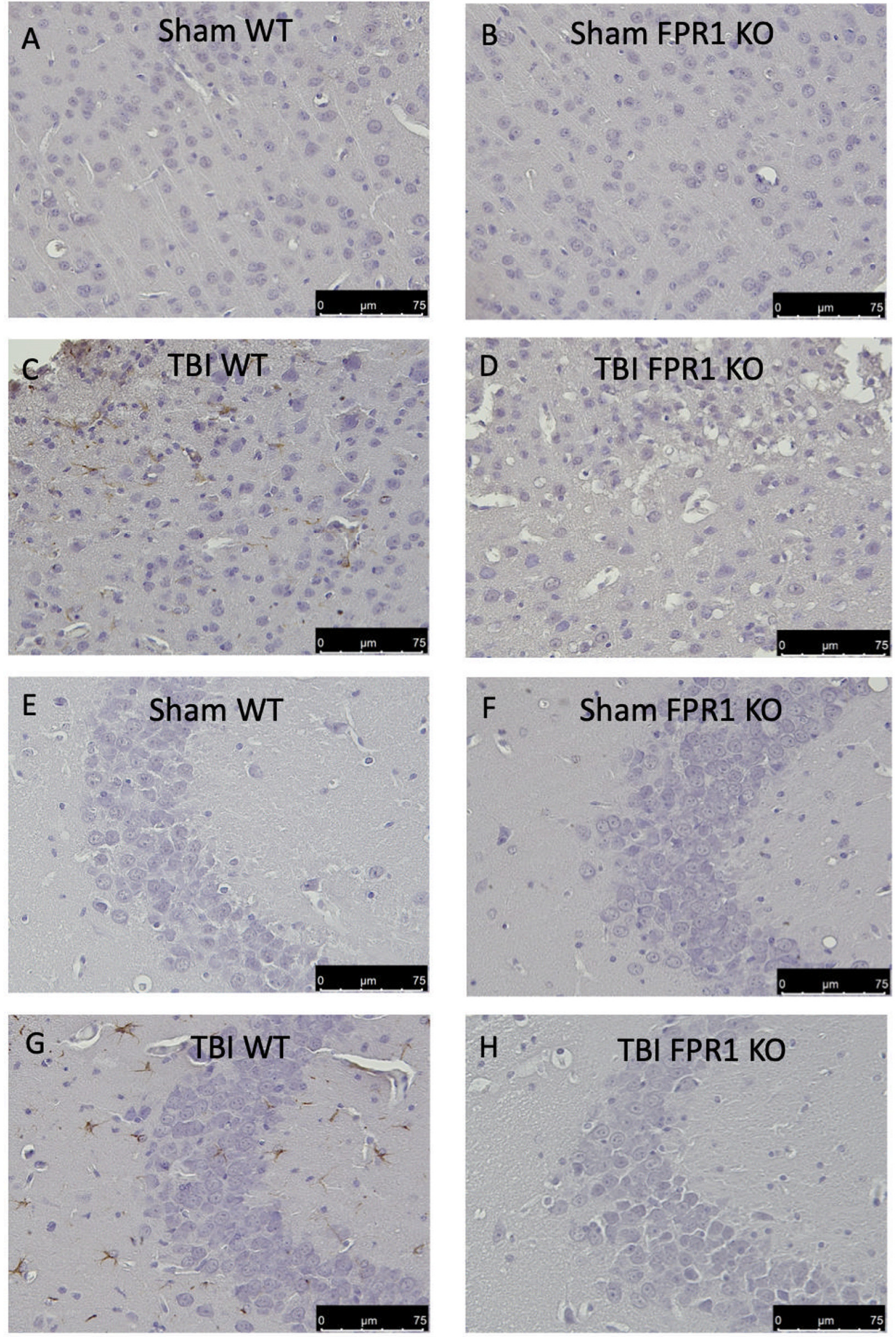

Figure 8. Effect of the absence of Fpr1 on astrocytes: Immunohistochemical analysis shows an upregulation of GFAP expression in the cortex (C) and hippocampus (G) in the TBI WT group $24 \mathrm{~h}$ after injury, compared to the Sham WT cortex (A) and hippocampus (E) and Sham Fpr1 KO cortex (B) and hippocampus (F) Fpr1 KO animals $24 \mathrm{~h}$ after TBI show reduced expression of GFAP-positive cells in both the cortex (D) and hippocampus (H) For immunohistochemical staining, a 40× magnification is shown $(75-\mu \mathrm{m}$ scale bar). For the analyses, $n=5$ animals from each group were employed. Results were analyzed by two-way ANOVA followed by a Bonferroni post-hoc test for multiple comparisons. Non-parametric data were analyzed with Fisher's exact test. scale bar $75 \mu \mathrm{m}$. 

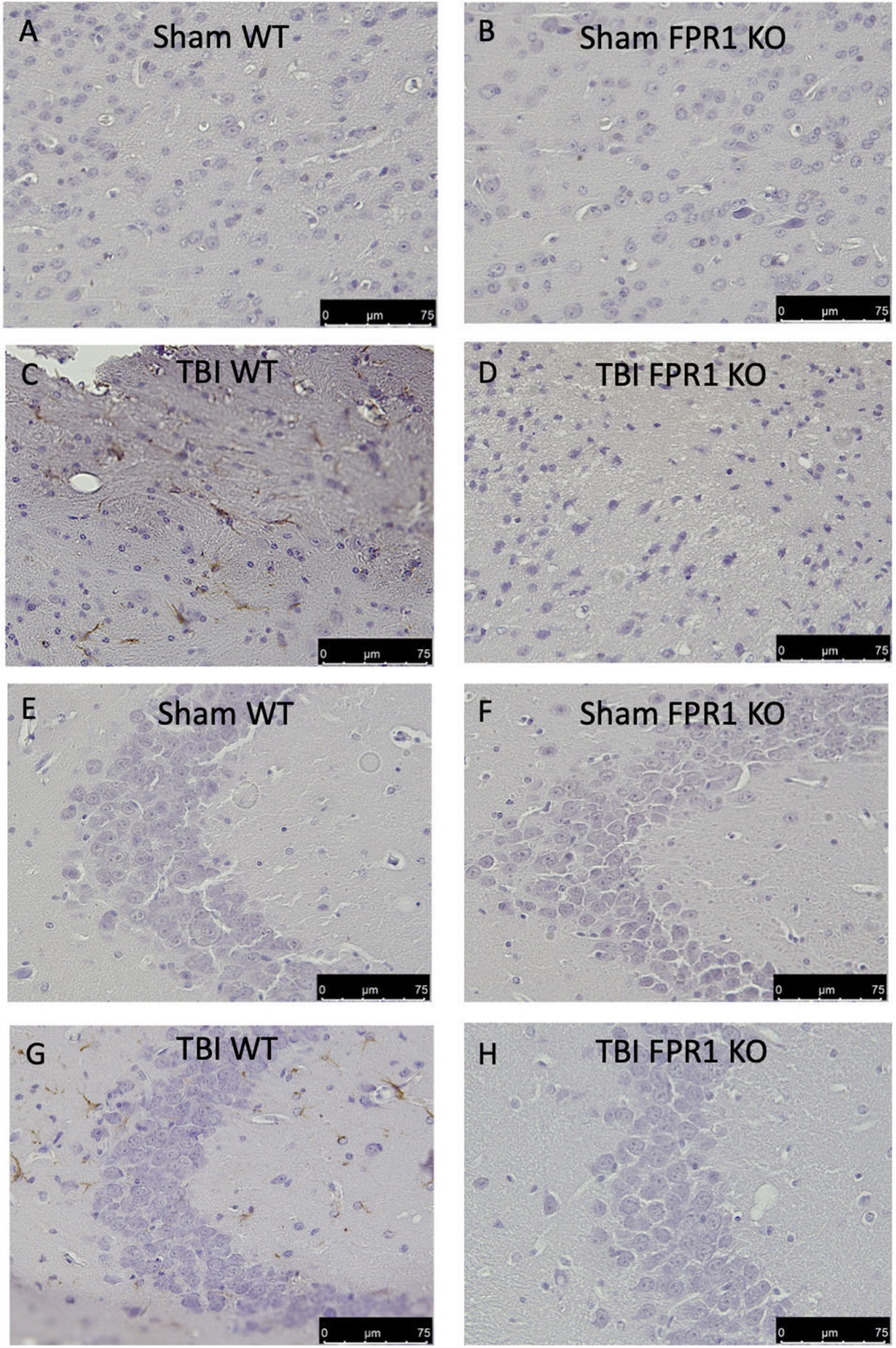

Figure 9. Effect of the absence of Fpr1 on microglia: Immunohistochemical analysis shows increased expression of Iba1 in TBI WT group cortex $(\mathbf{C})$ and hippocampus $(\mathbf{G})$, compared to the Sham WT cortex (A) and hippocampus (E) and Sham Fpr1 KO cortex (B) and hippocampus (F) Fpr1 KO animals $24 \mathrm{~h}$ after TBI show reduced expression of Iba1-positive cells in both the cortex (D) and hippocampus (H) For immunohistochemical staining, a $40 \times$ magnification is shown $(75-\mu \mathrm{m}$ scale bar). For the analyses, $n=5$ animals from each group were employed. Results were analyzed by two-way ANOVA followed by a Bonferroni post-hoc test for multiple comparisons. Non-parametric data were analyzed with Fisher's exact test. scale bar $75 \mu \mathrm{m}$. 

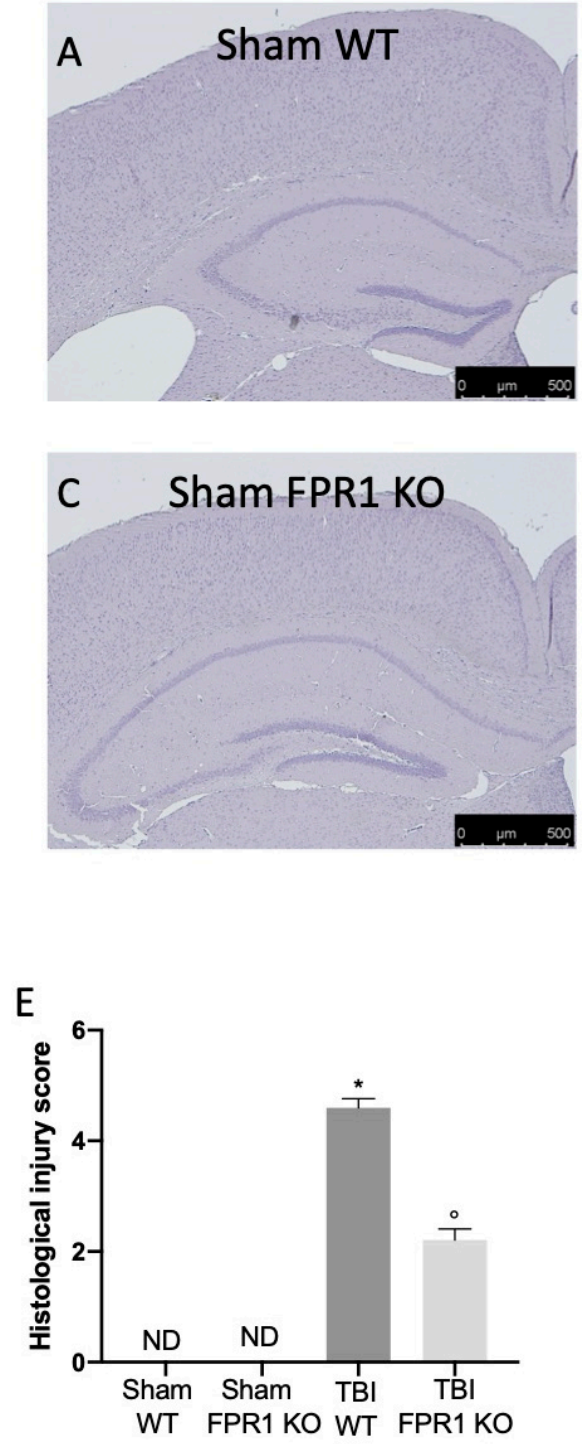
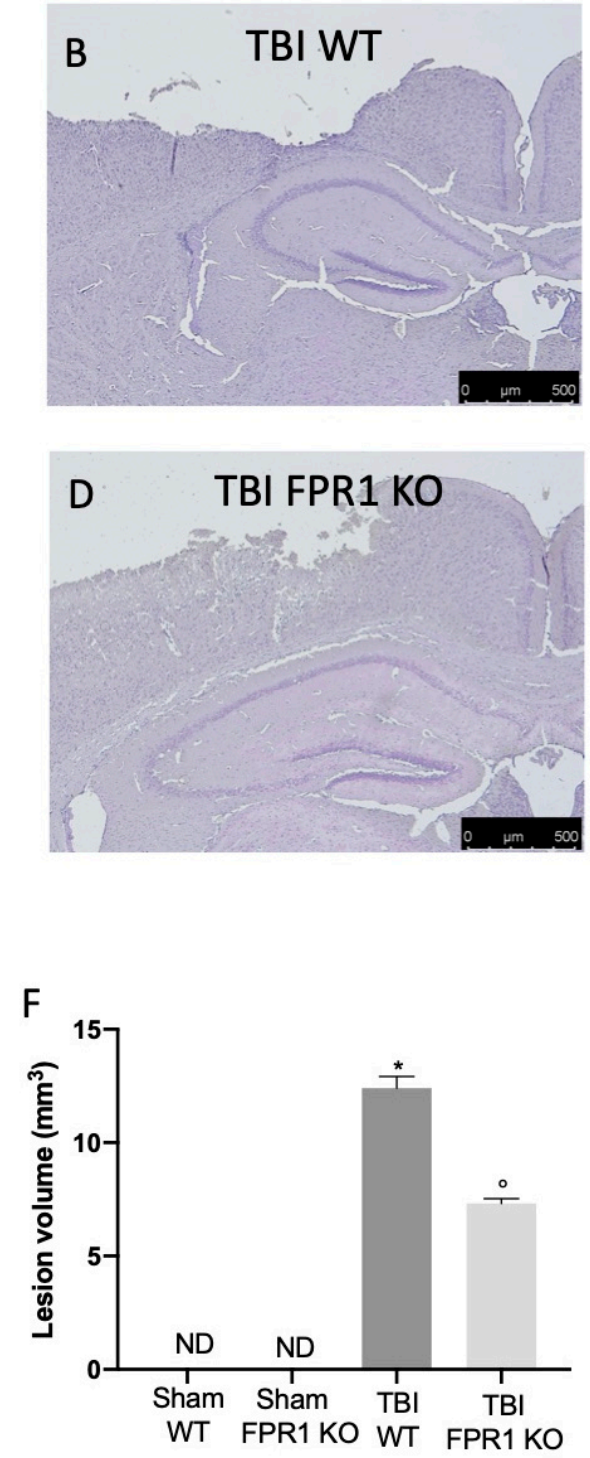

Figure 10. Effect of the absence of Fpr1 on histological alterations: No histological alterations were detected in brain section from mice of the Sham WT group (A) and Sham Fpr1 KO group (C). The TBI WT group four weeks after injury shows evident tissue inflammation (B). (D) Fpr1 KO animals subjected to TBI show less injury than the TBI WT group (E) Moreover, the TBI Fpr1 KO group displays a reduced lesion volume compared to the TBI WT group, as shown in (F) For the analyses, $n=5$ animals from each group were employed. Results were analyzed by two-way ANOVA followed by a Bonferroni post-hoc test for multiple comparisons. Non-parametric data were analyzed with Fisher's exact test. ${ }^{*} p<0.05$ vs. Sham WT; ${ }^{\circ} p<0.05$ vs. TBI WT. scale bar $500 \mu \mathrm{m}$.

3.11. Effect of Absence of Fpr1 on iNOS, COX-2 and Prostaglandin Expression Four Weeks after Traumatic Brain Injury

Immunohistochemical analysis showed increased COX-2 expression in TBI WT mice (Figure 11C) compared to the Sham WT (Figure 11A) and Fpr1 KO animals (Figure 11B), while TBI Fpr1 KO animals did not show any upregulation (Figure 11D). Western blot analysis of COX-2 confirmed this data (Figure 11E,F). Molecular analysis displayed increased expression of iNOS in TBI WT mice compared to the Sham WT and Fpr1 KO animals, while TBI Fpr1 KO animals did not show any upregulation (Figure 12A,D). Western blot analysis showed upregulated levels of PGE2 synthase (Figure 12B,E) in tissue samples collected from TBI WT mice compared to the Sham WT and Fpr1 KO animals, while TBI Fpr1 KO animals did not show increased levels. PGD2 synthase expression was downregulated in TBI 
WT animals, compared to the Sham WT and Fpr1 KO animals. In TBI Fpr1 KO animals, PGD2 synthase expression did not increase (Figure 12C,F).
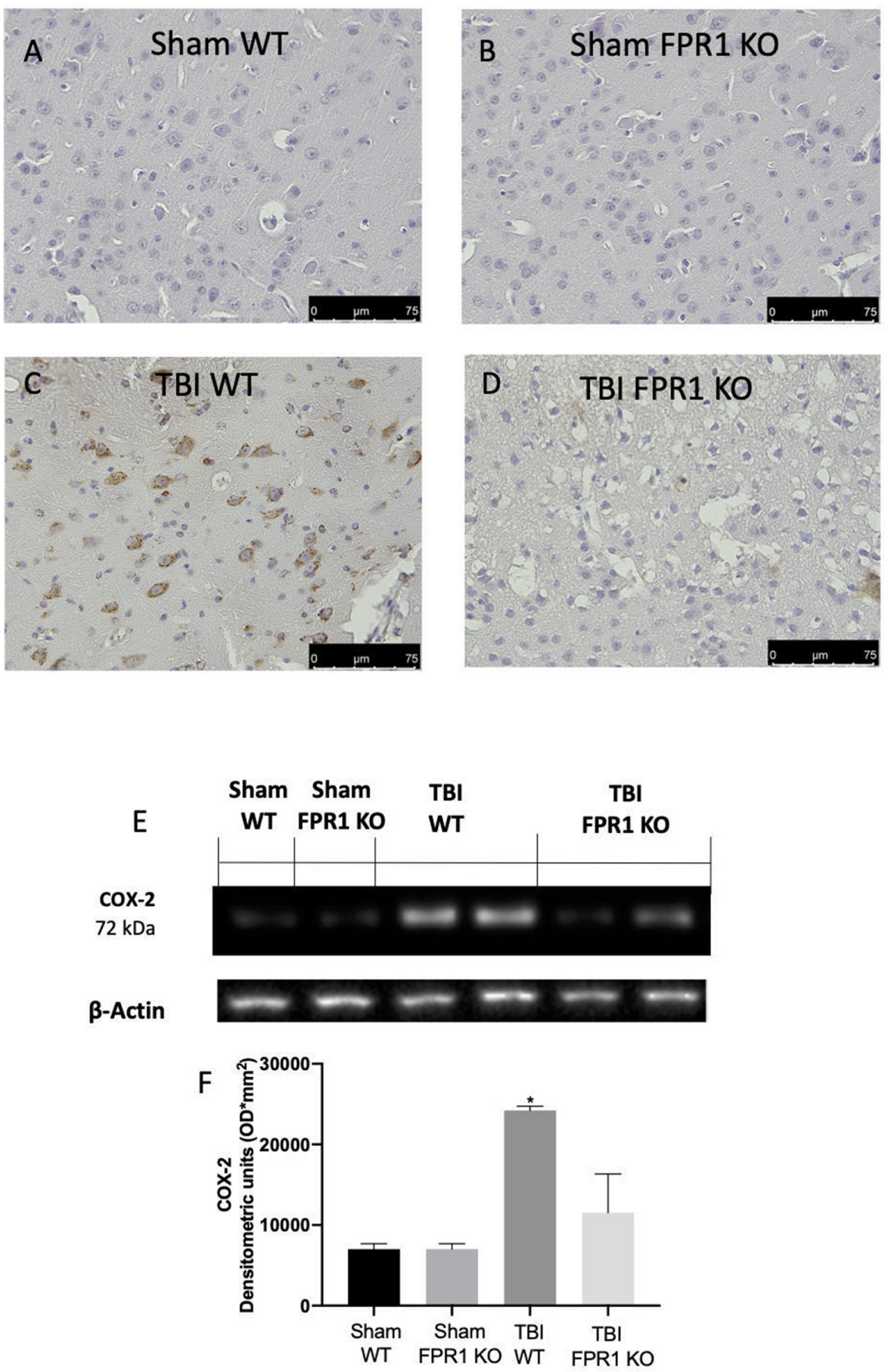

Figure 11. Effect of the absence of Fpr1 on COX-2 expression four weeks after TBI: Immunohistochemical analysis displays an increased COX-2 (C) expression in TBI WT mice compared to the Sham WT (A) and Fpr1 KO animals (B) while TBI Fpr1 KO animals do not show any increase (D) Western blot analysis confirmed the increase in COX-2 expression in TBI WT mice compared to the sham groups. COX-2 expression was significantly reduced in TBI Fpr1 KO animals (E,F) For the analyses, $n=5$ animals from each group were employed. Results were analyzed by two-way ANOVA followed by a Bonferroni post-hoc test for multiple comparisons. Non-parametric data were analyzed with Fisher's exact test. * $p<0.05$ vs. Sham WT. scale bar $75 \mu \mathrm{m}$. 

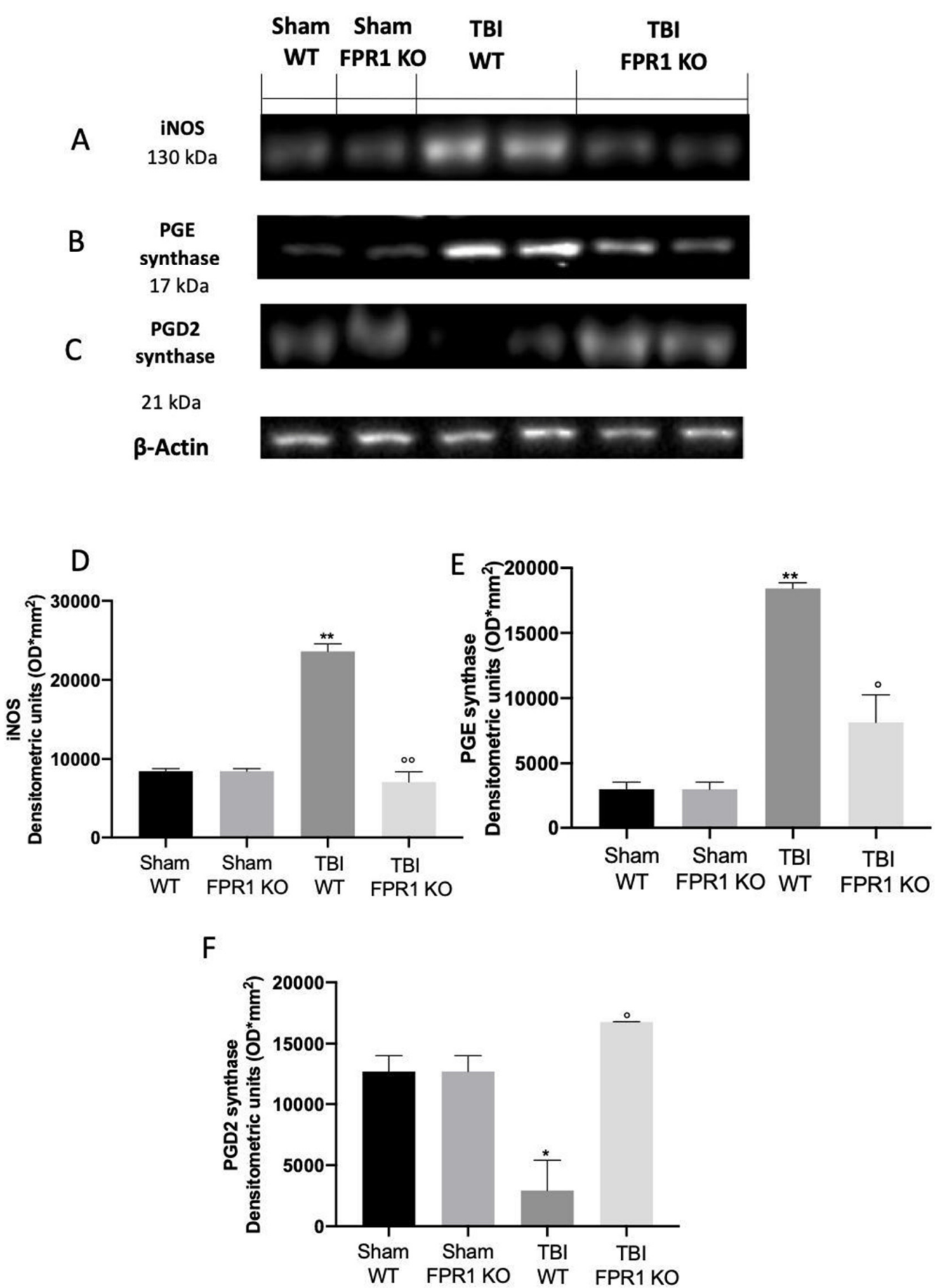

Figure 12. Effect of the absence of Fpr1 on iNOS, PGE2 and PGD2 synthase: Western blot analysis reveals increased iNOS levels in TBI WT mice compared to the Sham WT and Fpr1 KO animals (A) Tissues from TBI Fpr1 KO mice do not show any upregulation. Molecular analysis shows an increased PGE2 synthase (B) expression in TBI WT mice compared to the Sham WT and Fpr1 KO animals, while TBI Fpr1 KO animals do not show any increase. PGD2 synthase expression (C) decreases in WT animals four weeks after TBI, compared to the Sham WT and Fpr1 KO animals. In the TBI Fpr1 KO animals, PGD2 synthase expression remain at the basal levels; see the densitometric analysis (D-F) For the analyses, $n=5$ animals from each group were employed. Results were analyzed by two-way ANOVA followed by a Bonferroni post-hoc test for multiple comparisons. Non-parametric data were analyzed with Fisher's exact test. ${ }^{*} p<0.05$ vs. Sham WT; ${ }^{* *} p<0.01$ vs. Sham WT; ${ }^{\circ} p<0.05$ vs. TBI WT; ${ }^{\circ} p<0.01$ vs. TBI WT. 


\subsection{Effect of Absence of Fpr1 on Cell Proliferation Four Weeks after Traumatic Brain Injury}

BrdU $(50 \mathrm{mg} / \mathrm{Kg}$ ) was administered for seven days after the TBI, and then the mice were sacrificed after four weeks from the injury to label the proliferating neural progenitors in the DG. Sham WT and FPR1 KO animals showed the baseline of proliferating cells in the DG (Figure 13A,B,E). In the TBI WT group, the density of the surviving proliferated cells was elevated (Figure 13C,E) while the TBI Fpr1 KO group did not show any significant upregulation (Figure 13D,E).
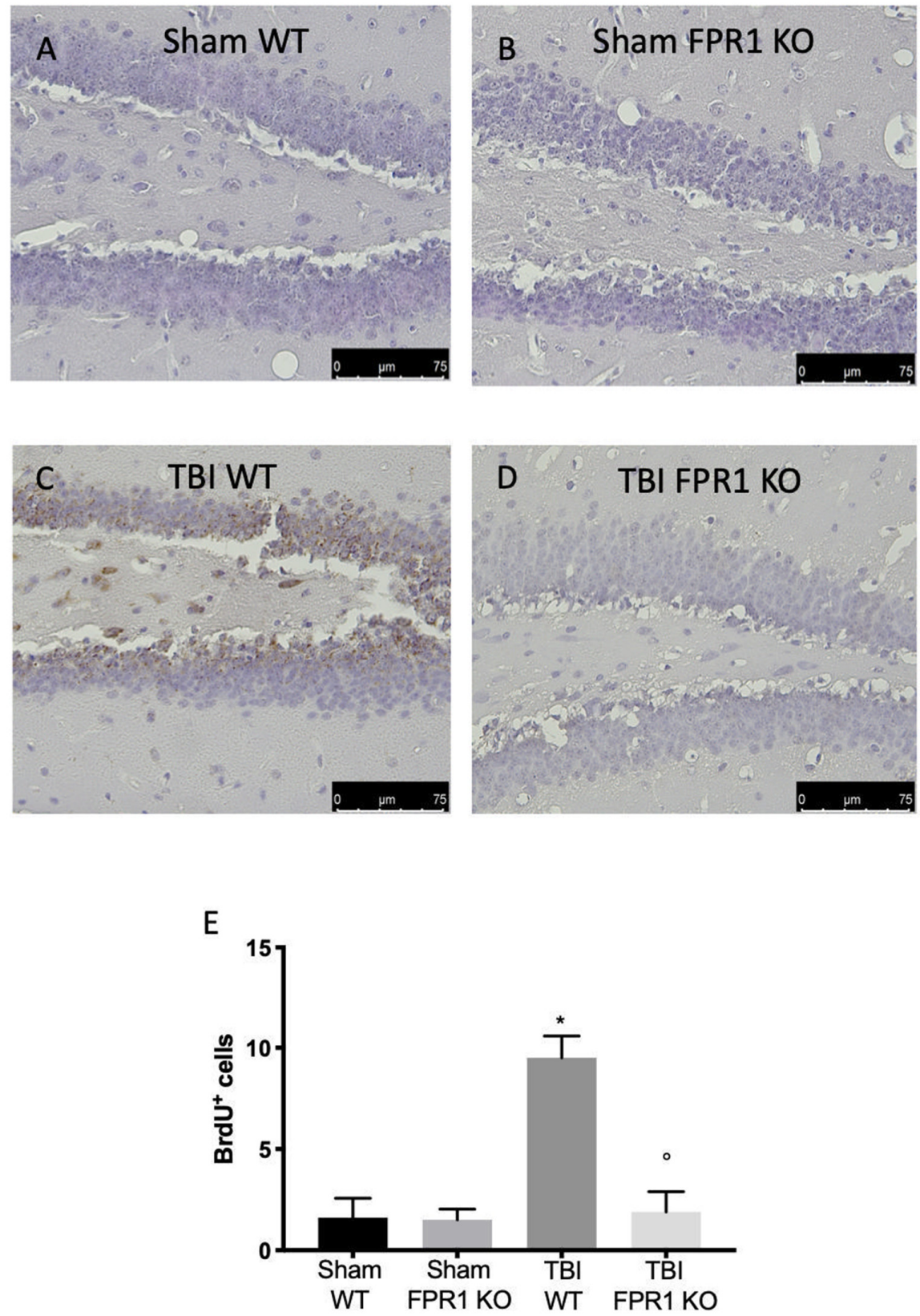

Figure 13. Effect of the absence of Fpr1 on neurogenesis: Immunohistochemical analysis shows an increased number of BrdU-positive proliferating cells in the Dentate Gyrus of WT animals subjected to TBI four weeks after injury (C) compared to the Sham WT group (A) and Sham Fpr1 KO group (B) Fpr1 $\mathrm{KO}$ animals four weeks after TBI shows significantly less BrdU-positive proliferating cells (D); see the densitometric analysis (E) For the analyses, $n=5$ animals from each group were employed. Results were analyzed by two-way ANOVA followed by a Bonferroni post-hoc test for multiple comparisons. Non-parametric data were analyzed with Fisher's exact test. ${ }^{*} p<0.05$ vs. Sham WT; ${ }^{\circ} p<0.05$ vs. TBI WT. scale bar $75 \mu \mathrm{m}$. 


\subsection{Effect of Absence of Fpr1 on Behavioral Performance Four Weeks after Traumatic Brain Injury}

Four weeks after traumatic brain injury induced by a controlled cortical impact, Morris water maze results showed defects in learning and memory abilities in TBI Fpr1 KO animals. During the training and the probe test, the TBI Fpr1 KO group took a longer time than the TBI WT group to find the hidden platform (Figure 14A,B). To further assess the locomotor activity in mutant mice, the Open Field test was performed. We found the number of crossings increased in TBI Fpr1 KO mice compared to the WT animals (Figure 14C,D); in contrast, the TBI Fpr1 KO group decreased time spent in the center. In order to evaluate impairments in their social interaction and exploring behavior, important clinical features of dementia, we performed the social interaction test and the novel object recognition test. Rodents have the natural habit of exploring new objects and interacting with other mice. In the social interaction test, we observed that the total duration of the contacts was decreased in the TBI Fpr1 KO animals compared to the TBI WT group, while the number of contacts was significantly increased in the TBI Fpr1 KO animals compared to the TBI WT group (Figure 14E,F). The novel object recognition test was used to evaluate changes in cognitive function. Fpr1 $\mathrm{KO}$ animals subjected to traumatic brain injury had less exploratory behavior and spent significantly less time with the novel object, indicating compromise of cognitive function, while in TBI WT mice the function within the novel object recognition test returned to normal values (Figure 14G).

\subsection{Effect of Absence of Fpr1 on Self-Renewal and Neurogenesis Four Weeks after Traumatic Brain Injury}

To determine whether the absence of the Fpr1 receptor influence the neuronal differentiation, four weeks after traumatic brain injury a Western blot analysis for p-AKT, AKT, $\beta$-III tubulin and GFAP was performed. The Western blot showed that the p-AKT/AKT ratio significantly increased in WT animals four weeks after injury, compared to the TBI Fpr1 KO animals (Figure 15A,D). In WT animals subjected to TBI, the expression of the neuron marker $\beta$-III tubulin also increased (Figure 15B,E); meanwhile, levels of glial fibrillary acidic protein (GFAP) gradually decreased (Figure 15C,F). Four weeks after the TBI, in the absence of Fpr1 $\beta$-III, the tubulin levels did not increase (Figure 15B,E), while the GFAP levels were significantly upregulated compared to the WT animals (Figure 15C,F). 

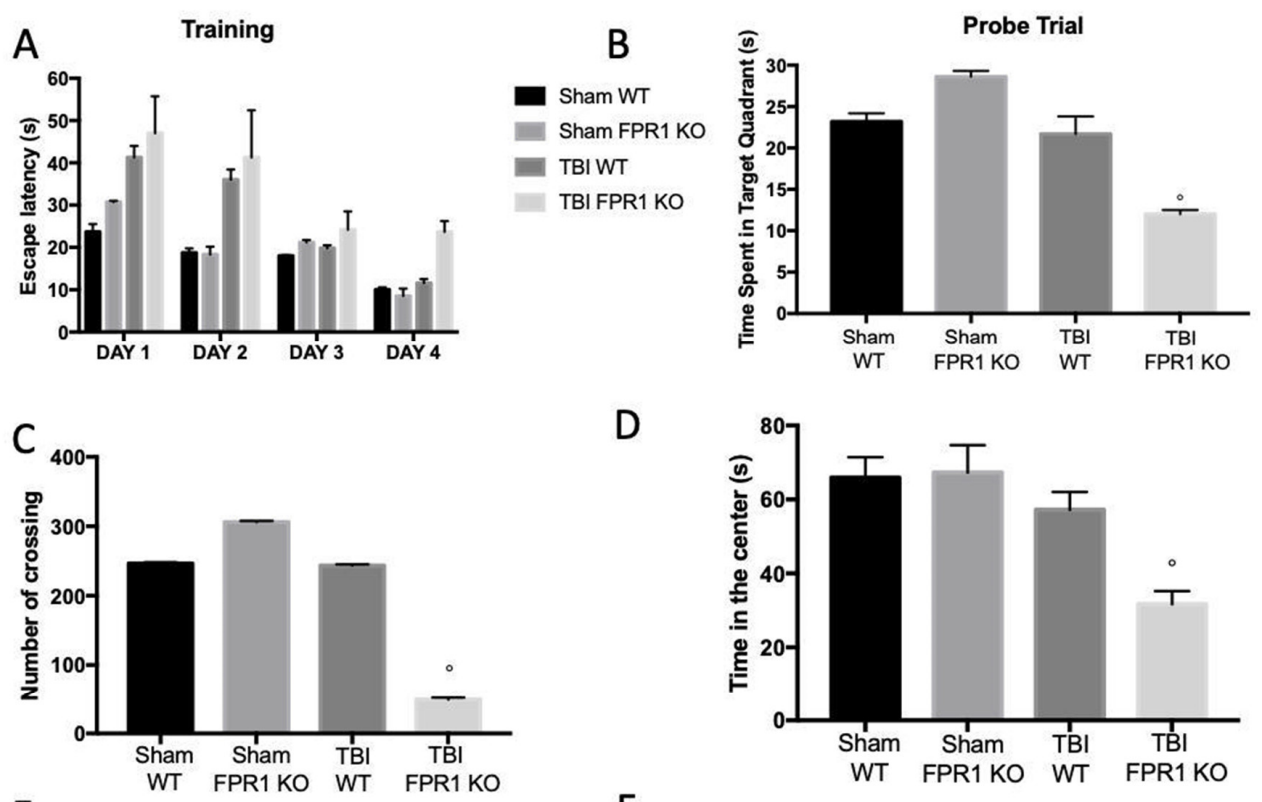

D
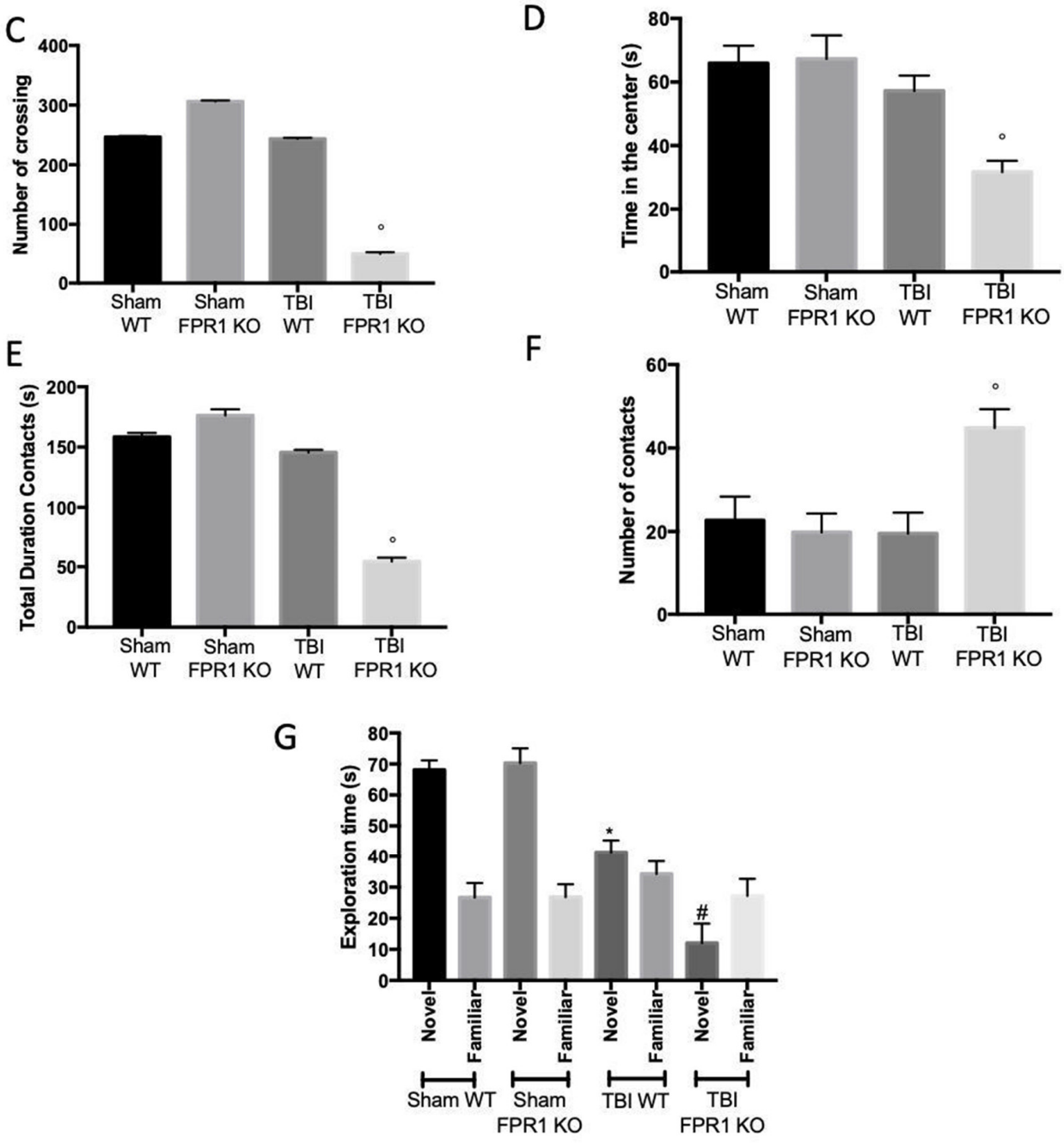

Figure 14. Effect of the absence of Fpr1 on behavioral tests: Training and Probe trial of the Morris water maze performed four weeks after injury shows that the TBI Fpr1 KO group takes a longer time than the TBI WT group to find the hidden platform $(\mathbf{A}, \mathbf{B})$ In the Elevated Plus Maze test performed four weeks after injury, the TBI Fpr1 KO mice spend significantly less time in the open arm and in the central zone than WT animals (C,D). In the social interaction test we observed that the total duration of the contacts was decreased in the TBI Fpr1 KO animals compared to the TBI WT group, while the number of contacts was significantly increased in the TBI Fpr1 KO animals compared to the TBI WT group (E,F) In the novel object recognition test, the Fpr1 $\mathrm{KO}$ animals subjected to traumatic brain injury spend significantly less time with the novel object, while in the TBI WT mice the function within the novel object recognition test returned to normal values $(\mathbf{G})$. For the analyses, $n=5$ animals from each group were employed. Results were analyzed by two-way ANOVA followed by a Bonferroni post-hoc test for multiple comparisons. Non-parametric data were analyzed with Fisher's exact test. ${ }^{*} p<0.05$ vs. Sham WT; ${ }^{\circ} p<0.05$ vs. TBI WT; ${ }^{\#} p<0.05$ vs. TBI WT. 

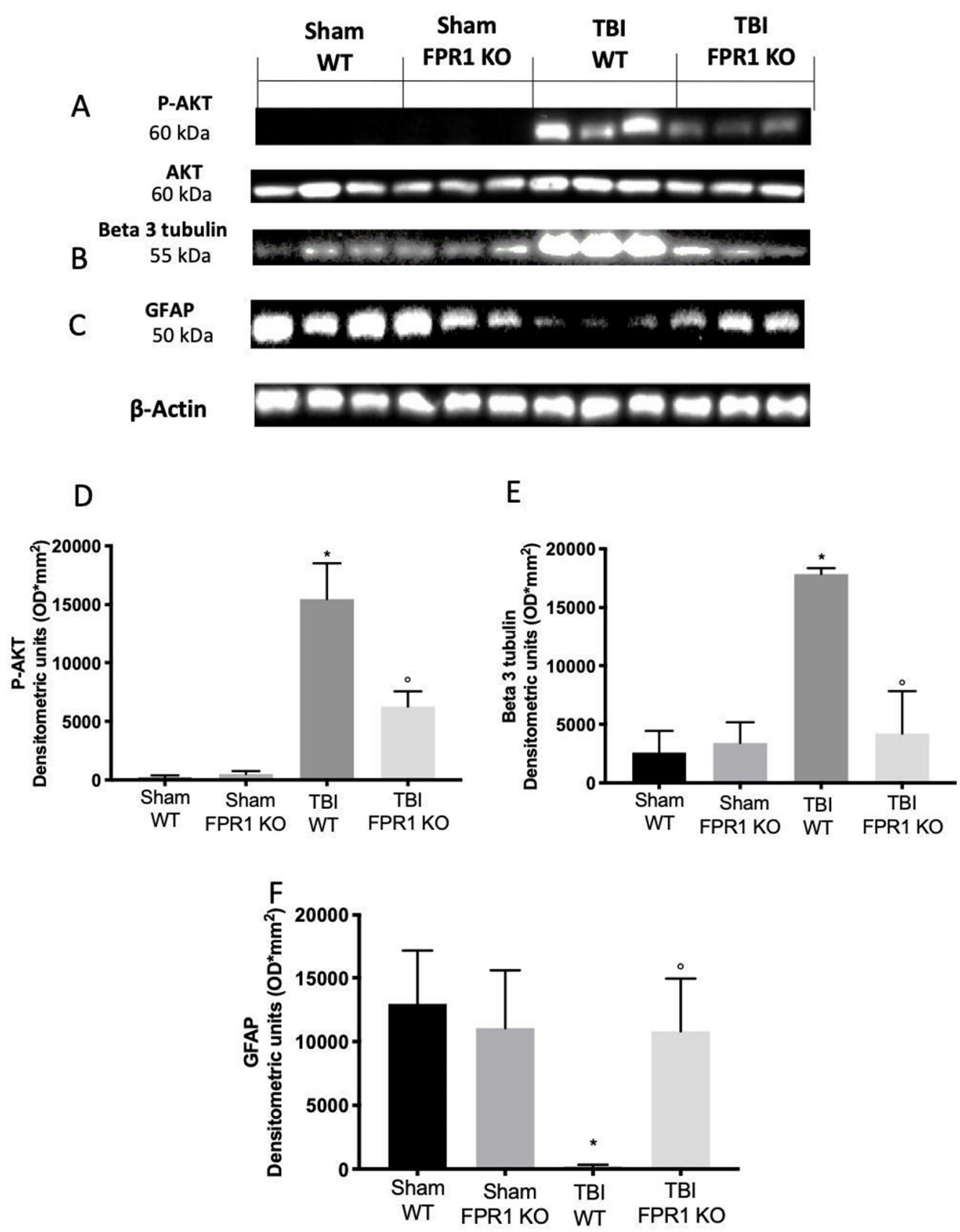

Figure 15. Effect of the absence of Fpr1 on neuronal differentiation: Western blot analysis shows an upregulation of p-AKT (A) and $\beta$ 3-tubulin (B) expression in the TBI WT group four weeks after injury, compared to the Sham WT and Sham Fpr1 KO group. Fpr1 KO animals four weeks after TBI show reduced levels of $\mathrm{p}$-AKT and $\beta$ 3-tubulin compared to the TBI WT group; see the densitometric analysis $(\mathbf{D}, \mathbf{E})$ The same analysis shows basal expression of GFAP (C) in the Sham WT group and Sham Fpr1 KO group. A significant reduction in GFAP expression is expressed in the TBI WT group four weeks after injury, while TBI Fpr1 KO animals show a restored expression of the Glial Fibrillary Acidic Protein expression; see the densitometric analysis (F) For the analyses, $n=5$ animals from each group were employed. Results were analyzed by two-way ANOVA followed by a Bonferroni post-hoc test for multiple comparisons. Non-parametric data were analyzed with Fisher's exact test. ${ }^{*} p<0.05$ vs. Sham WT; ${ }^{\circ} p<0.05$ vs. TBI WT. 


\section{Discussion}

In this study, we analyzed the effect of the Fpr1 gene deletion on animals subjected to traumatic brain injury, from the early stage of acute inflammation to the neurogenesis four weeks from the injury. Controlled cortical impact is one of the most used animal models to induce traumatic brain injury thanks to its ability to reproduce what happens in humans [52]. Brain trauma induces tissue injury, release of inflammatory mediators and alteration of the blood brain barrier, which, in turn, allows neutrophils, macrophages and lymphocytes to access to lesion site [53]. Once recruited, inflammatory cells are activated and triggers multiple pathways, such as increasing gene transcription, assembly of intracellular pro-inflammatory complexes, release of reactive oxygen species and nitric oxide [54]. Neutrophils are one of the most important cells in the inflammatory response in the central nervous system (CNS) after traumatic brain injury $[55,56]$. Fpr1 are constitutively expressed on quiescent neutrophils and rapidly upregulated in response to inflammatory stimuli. The important pathogenic role of Fpr1 has been already described in experimental colitis and endometriosis, showing modulation of immune cell recruitment together with a modulation of local cellular activation and survival $[50,57]$. In particular, Fpr1 synthesis happens late in neutrophil maturation, with storage in azurophilic granules and secretory vesicles [58,59]. Neutrophil activation induces Fpr1 translocation on cell surfaces and upregulates Fpr1 protein synthesis. Once activated, as G-protein-coupled receptors, Fpr1 induces guanosine diphosphate (GDP) transformation into guanosine triphosphate (GTP). GTP catalyzes the dissociation of $\alpha$ from the $\beta \gamma$ subunits, stimulating phosphoinositide 3-kinase $\gamma$ (PI3K $\gamma$ ) phospholipase $C \beta$ (PLC $\beta$ ) and the MAPK signaling pathway $[50,60,61]$. The first part of the study demonstrates the beneficial effect of Fpr1 gene deletion in acute response in a model of cerebral trauma. Twenty-four hours after traumatic brain injury, the myeloperoxidase (MPO) test, used as a specific and sensitive method to quantify neutrophil accumulation, display a reduction in MPO activity in animals lacking the Fpr1 gene, compared to the WT animals [62]. In particular, Fpr1 KO mice showed reduced infiltration of neutrophils, macrophages and immune cells to the site of injury. In absence of the Fpr1 gene, the second messengers p38 MAPK and the extracellular signal-regulated kinase (ERK), normally increased twenty-four hours after injury [63], were not phosphorylated. Fpr1 gene deletion display a positive effect against trauma through the inhibition of the MAPK pathway.

The ERK1/2, PI-3K and p38 MAPK signaling pathways are directly involved in the induction of COX-2 in neutrophils [64]. After brain injury, the COX 2 gene expression levels and PGE2 synthase increased while the PGD2 synthase decreased, suggesting that PGE2 and PGD2 afforded contraindicative effects of inflammation and anti-inflammation, respectively [65]. In the absence of Fpr1, the COX-2 levels were decreased, as was PGE2 synthase, while PGD2 synthase showed increased levels compared to the WT animals, indicating that Fpr1 could modulate acute inflammation acting through the COX-2 pathway.

PLC $\beta$ hydrolyses phosphoinositol-4,5-bisphosphate ( $\left.\mathrm{PIP}_{2}\right)$ into inositol 1,4,5-trisphosphate $\left(\mathrm{IP}_{3}\right)$ and diacylglycerol (DAG) [11]. $\mathrm{IP}_{3}$ induces calcium release from the endoplasmic reticulum. An increase in calcium concentration triggers the calmodulin $(\mathrm{CaM}) /$ calcineurin pathway. DAG remains localized on the membrane and mediates the association of protein kinase C (PKC) to the cell membrane, starting a chain of phosphorylation, which will contribute to the functional responses of the cell. $\mathrm{PKC}$ isoforms are responsible of the transcription factor nuclear factor kappa B (NF- $\mathrm{KB}$ ) translocation to the nucleus. NF-kB is involved in different cellular processes, such as cell proliferation, oxidative stress apoptosis and secretion of cytokines [66]. Usually, it is bound by the inhibitor protein IkB $\alpha$ into the cytoplasm. Several stimuli, including the brain tissue injury, induce the degradation of $\operatorname{IkB} \alpha$, freeing NF-kB. The positive effects of the Fpr1 gene deletion may be attributed, in part, to the downregulation of the NF-kB pathway. Brain tissues collected from Fpr1 KO mice showed a reduced IkB $\alpha$ degradation into the cytoplasm and NF- $\mathrm{kB}$ translocation into the nucleus induced by the injury. Through this inhibition, the absence of Fpr1 reduces the transcription of the NF-KB target genes involved in the enhancement of the inflammatory process, such as the NLRP3 inflammasome. 
Recently, the master role of the NLRP3 inflammasome in the inflammatory response to a traumatic brain injury has been underlined $[36,67,68]$; its inhibition can ameliorate the secondary events associated with traumatic brain injury [69]. NLRP3 inflammasome is a multiprotein complex, constituted by an adaptor protein containing a caspase-recruitment domain (ASC) and the serine protease caspase 1 (Casp1) [70]. The assembly of this complex catalyzes the activation of Casp1, which in turn cleaves and activates the pro-inflammatory cytokines IL-1 $\beta$ and IL-18, supporting the inflammatory process. Once again, the Frp1 gene deletion may reduce the NLRP3 transcription and the resulting assembly of the inflammasome complex by reducing the activation of the acute phase of inflammation.

Moreover, the upregulation of proinflammatory cytokines induces generation of free oxygen radicals, immune cells proteases and toxic metabolites [71,72]. Generation of ROS lead to oxidative stress, causing alteration of several signaling pathways [36]. One effect of the increased oxidative stress is the modulation of the redox-sensitive protein expression [73]. In our study, we underlined that the absence of the Fpr1 gene expression stabilized the levels of the anti-oxidant enzyme Mn-SOD induced by traumatic brain injury. Therefore, the upregulation of iNOS expression can enhance the oxidative stress associated with brain injury [74,75] Fpr1 KO animals displayed a reduced iNOS expression. After TBI, the accumulation of circulating macrophages, neutrophils, lymphocytes and the release of inflammatory mediators to the lesion site activates the glial and inflammatory cells, thus supporting the inflammatory process [53,76-78]. Our results show that the absence of Fpr1 gene expression leads to a reduction of Iba1 and GFAP expression at the early phase of inflammation. Interestingly, the major pro-inflammatory markers continue to be overexpressed in the hippocampus and brain cortex, even four weeks after a traumatic brain injury [79], while mice lacking the Fpr1 gene showed a reduction of neuroinflammation compared to the WT animals. In order to evaluate the inflammatory course four weeks after traumatic brain injury, we evaluated several inflammatory markers also at this timepoint.

Neuroinflammation and oxidative stress were significantly increased at this time point $[80,81]$. Our data confirmed the persistent inflammation four weeks after injury in WT animals, while the absence of the Fpr1 showed a reduction in oxidative stress, cyclooxygenase-2 and prostaglandin synthase expression. It is well described that TBI induces secondary processes, leading to neurological disorders, such as learning and memory impairment [26]. In contrast, four weeks after injury, severe controlled cortical impact significantly promoted neuron differentiation and cell proliferation [82]. In particular, it promotes neurogenesis at three stages: immature neurons, NSC proliferation and newly-generated mature neurons [28,29,82]. Neural stem cells care for adult neurogenesis and represent the regenerative potential of the brain through a lifetime. This proliferation after traumatic brain injury brings about the possibility of repair injury by controlled cortical impact. Recently, Fprs have been found in stem cells [83-85], the spinal cord, human brain, hypoglossal nucleus neurons and anterior horn cells [12]. Moreover, protein levels of Fpr1 increased during the differentiation process in the neural stem cells [27]. In particular, neural stem cells can differentiate into astrocytes, neurons and oligodendrocytes, expressing GFAP, beta-III tubulin and Olig2 [86]. The second part of the study evaluated the effect of the absence of the Fpr1 gene expression on neurological disorders and neurogenesis associated with the traumatic brain injury. The number of BrdU-incorporating cells showed an increased neurogenesis in WT animals subjected to TBI four weeks after injury compared to the Fpr1 KO animals. To address whether the observed difference between the Fpr1 KO mice and WT mice was also shown in animal behavior, several tests were performed. Behavioral tests showed the impaired cognitive and social functions of the Fpr1 KO mice subjected to TBI compared to the WT animals with the same lesion. In particular, in the Morris water maze test, Fpr1 KO animals displayed reduced memory and learning capacity; moreover, in the Open Field, social interaction and novel object recognition tests, they showed reduced exploratory activity and anxiety-like behavior.

To further comprehend the mechanism by which Fpr1 influenced neurogenesis, the molecules associated with neuronal differentiation were assessed. A recent study underlined that self-renewal and neurogenesis are regulated through the PI3K/Akt pathway [87]. In this work, for the first time 
we show that, four weeks after traumatic brain injury, neurogenesis and neuronal differentiation are activated by Fpr1 signaling. This pathway involves PI3K upregulation and AKT phosphorylation. In the absence of Fpr1 gene expression, animals subjected to traumatic brain injury displayed reduced neural differentiation while showing upregulation of astrocyte differentiation.

\section{Conclusions}

Twenty-four hours after traumatic brain injury, the absence of the Fpr1 gene showed a positive effect by reducing the acute inflammation. In particular, we observed the reduction of the p-38 and ERK phosphorylation, a reduced expression of NFkB into the nucleus and a reduced expression of the components of the NLRP3 inflammasome complex, which also led to a reduction in the levels of IL-1beta and IL-18. We investigated iNOS, COX-2, PGE synthase and PGD2 synthase expression levels at $24 \mathrm{~h}$ and a chronic timepoint as a marker of inflammation. The positive effects of the absence of Fpr1 gene expression on inflammation are due to its priming effect of the inflammatory pathway. In our experiment, four weeks after injury, the severe controlled cortical impact significantly promoted neuron differentiation and cell proliferation. Behavioral tests showed the impaired cognitive and social functions of the Fpr1 KO mice subjected to TBI compared to the WT animals with the same lesion. From a molecular point of view, self-renewal and neurogenesis are regulated through the PI3K/Akt pathway. It was found that the activation of FPRs promoted the neuronal differentiation of neural stem cells and inhibited their differentiation into astrocytes, and this process was dependent on the PI3K-AKT signaling pathway. We showed that in the absence of the Fpr1 gene expression a reduced AKT phosphorylation was observed. This led to a reduced differentiation into the neurons, showed by a reduction in beta-III tubulin expression, while GFAP expression was increased, showing no inhibition of astrocyte differentiation. Collectively, our study reported that the absence of Fpr1 gene expression reduced inflammation and oxidative stress immediately after traumatic brain injury and at a chronic timepoint. Four weeks after traumatic brain injury, Fpr1 promoted the differentiation of neuronal stem cells into neurons and reduced their differentiation into astrocytes via the PI3K/Akt pathway.

Supplementary Materials: The following are available online at http://www.mdpi.com/2079-7737/9/9/238/s1, Figure S1: The whole western blots.

Author Contributions: Conceptualization, S.C. and R.D.P.; methodology, D.I., M.S., M.E., A.P.; software, M.C.; validation, M.C., R.S. and E.G.; formal analysis, R.C.; investigation, A.F.P.; resources, R.F.; data curation, R.D.; writing — original draft preparation, R.F.; writing—review and editing, R.D.P.; visualization, E.G.; supervision, R.D.P.; project administration, S.C.; funding acquisition, S.C. All authors have read and agreed to the published version of the manuscript.

Funding: This research received no external funding.

Conflicts of Interest: The authors declare no conflict of interest.

\section{References}

1. Dahlgren, C.; Gabl, M.; Holdfeldt, A.; Winther, M.; Forsman, H. Basic characteristics of the neutrophil receptors that recognize formylated peptides, a danger-associated molecular pattern generated by bacteria and mitochondria. Biochem. Pharmacol. 2016, 114, 22-39. [CrossRef] [PubMed]

2. Ye, R.D.; Boulay, F.; Wang, J.M.; Dahlgren, C.; Gerard, C.; Parmentier, M.; Serhan, C.N.; Murphy, P.M. International Union of Basic and Clinical Pharmacology. LXXIII. Nomenclature for the formyl peptide receptor (FPR) family. Pharmacol. Rev. 2009, 61, 119-161. [CrossRef] [PubMed]

3. Spurr, L.; Nadkarni, S.; Pederzoli-Ribeil, M.; Goulding, N.J.; Perretti, M.; D' Acquisto, F. Comparative analysis of Annexin A1-formyl peptide receptor 2/ALX expression in human leukocyte subsets. Int. Immunopharmacol. 2011, 11, 55-66. [CrossRef] [PubMed]

4. Yang, D.; Chen, Q.; Le, Y.; Wang, J.M.; Oppenheim, J.J. Differential regulation of formyl peptide receptor-like 1 expression during the differentiation of monocytes to dendritic cells and macrophages. J. Immunol. 2001, 166, 4092-4098. [CrossRef] 
5. El Kebir, D.; Jozsef, L.; Filep, J.G. Opposing regulation of neutrophil apoptosis through the formyl peptide receptor-like 1/lipoxin A4 receptor: Implications for resolution of inflammation. J. Leukoc. Biol. 2008, 84, 600-606. [CrossRef]

6. Yang, D.; Chen, Q.; Gertz, B.; He, R.; Phulsuksombati, M.; Ye, R.D.; Oppenheim, J.J. Human dendritic cells express functional formyl peptide receptor-like-2 (FPRL2) throughout maturation. J. Leukoc. Biol. 2002, 72 , 598-607.

7. Gemperle, C.; Schmid, M.; Herova, M.; Marti-Jaun, J.; Wuest, S.J.; Loretz, C.; Hersberger, M. Regulation of the formyl peptide receptor 1 (FPR1) gene in primary human macrophages. PLoS ONE 2012, 7, e50195. [CrossRef]

8. Keitoku, M.; Kohzuki, M.; Katoh, H.; Funakoshi, M.; Suzuki, S.; Takeuchi, M.; Karibe, A.; Horiguchi, S.; Watanabe, J.; Satoh, S.; et al. FMLP actions and its binding sites in isolated human coronary arteries. J. Mol. Cell Cardiol. 1997, 29, 881-894. [CrossRef]

9. Heo, S.C.; Kwon, Y.W.; Jang, I.H.; Jeong, G.O.; Yoon, J.W.; Kim, C.D.; Kwon, S.M.; Bae, Y.S.; Kim, J.H. WKYMVm-induced activation of formyl peptide receptor 2 stimulates ischemic neovasculogenesis by promoting homing of endothelial colony-forming cells. Stem Cells 2014, 32, 779-790. [CrossRef]

10. Anton, P.; O'Connell, J.; O'Connell, D.; Whitaker, L.; O'Sullivan, G.C.; Collins, J.K.; Shanahan, F. Mucosal subepithelial binding sites for the bacterial chemotactic peptide, formyl-methionyl-leucyl-phenylalanine (FMLP). Gut 1998, 42, 374-379. [CrossRef]

11. Babbin, B.A.; Jesaitis, A.J.; Ivanov, A.I.; Kelly, D.; Laukoetter, M.; Nava, P.; Parkos, C.A.; Nusrat, A. Formyl peptide receptor-1 activation enhances intestinal epithelial cell restitution through phosphatidylinositol 3-kinase-dependent activation of Rac1 and Cdc42. J. Immunol. 2007, 179, 8112-8121. [CrossRef] [PubMed]

12. Becker, E.L.; Forouhar, F.A.; Grunnet, M.L.; Boulay, F.; Tardif, M.; Bormann, B.J.; Sodja, D.; Ye, R.D.; Woska, J.R., Jr.; Murphy, P.M. Broad immunocytochemical localization of the formylpeptide receptor in human organs, tissues, and cells. Cell Tissue Res. 1998, 292, 129-135. [CrossRef]

13. Chen, K.; Iribarren, P.; Huang, J.; Zhang, L.; Gong, W.; Cho, E.H.; Lockett, S.; Dunlop, N.M.; Wang, J.M. Induction of the formyl peptide receptor 2 in microglia by IFN-gamma and synergy with CD40 ligand. J. Immunol. 2007, 178, 1759-1766. [CrossRef] [PubMed]

14. Cui, Y.H.; Le, Y.; Zhang, X.; Gong, W.; Abe, K.; Sun, R.; Van Damme, J.; Proost, P.; Wang, J.M. Up-regulation of FPR2, a chemotactic receptor for amyloid beta 1-42 (A beta 42), in murine microglial cells by TNF alpha. Neurobiol. Dis. 2002, 10, 366-377. [CrossRef]

15. Cui, Y.H.; Le, Y.; Gong, W.; Proost, P.; Van Damme, J.; Murphy, W.J.; Wang, J.M. Bacterial lipopolysaccharide selectively up-regulates the function of the chemotactic peptide receptor formyl peptide receptor 2 in murine microglial cells. J. Immunol. 2002, 168, 434-442. [CrossRef]

16. Peeters, W.; van den Brande, R.; Polinder, S.; Brazinova, A.; Steyerberg, E.W.; Lingsma, H.F.; Maas, A.I. Epidemiology of traumatic brain injury in Europe. Acta Neurochir. 2015, 157, 1683-1696. [CrossRef]

17. Cheng, G.; Kong, R.H.; Zhang, L.M.; Zhang, J.N. Mitochondria in traumatic brain injury and mitochondrialtargeted multipotential therapeutic strategies. Br. J. Pharmacol. 2012, 167, 699-719. [CrossRef]

18. Wang, L.; Shi, J.X.; Yin, H.X.; Ma, C.Y.; Zhang, Q.R. The influence of subarachnoid hemorrhage on neurons: An animal model. Ann. Clin. Lab. Sci. 2005, 35, 79-85.

19. Chesnut, R.M.; Marshall, L.F.; Klauber, M.R.; Blunt, B.A.; Baldwin, N.; Eisenberg, H.M.; Jane, J.A.; Marmarou, A.; Foulkes, M.A. The role of secondary brain injury in determining outcome from severe head injury. J. Trauma 1993, 34, 216-222. [CrossRef]

20. Polinder, S.; Haagsma, J.A.; van Klaveren, D.; Steyerberg, E.W.; Van Beeck, E.F. Health-related quality of life after TBI: A systematic review of study design, instruments, measurement properties, and outcome. Popul. Health Metr. 2015, 13, 4. [CrossRef]

21. Hall, E.D.; Sullivan, P.G.; Gibson, T.R.; Pavel, K.M.; Thompson, B.M.; Scheff, S.W. Spatial and temporal characteristics of neurodegeneration after controlled cortical impact in mice: More than a focal brain injury. J. Neurotrauma 2005, 22, 252-265. [CrossRef]

22. Guevara, A.B.; Démonet, J.-F.; Polejaeva, E.; Knutson, K.M.; Wassermann, E.M.; Krueger, F.; Grafman, J. Association between long-term cognitive decline in Vietnam Veterans with TBI and caregiver attachment style. J. Head Trauma Rehabil. 2015, 30, E26-E33. [CrossRef] 
23. Coughlin, J.M.; Wang, Y.; Munro, C.A.; Ma, S.; Yue, C.; Chen, S.; Airan, R.; Kim, P.K.; Adams, A.V.; Garcia, C. Neuroinflammation and brain atrophy in former NFL players: An in vivo multimodal imaging pilot study. Neurobiol. Dis. 2015, 74, 58-65. [CrossRef]

24. Irrera, N.; Pizzino, G.; Calò, M.; Pallio, G.; Mannino, F.; Famà, F.; Arcoraci, V.; Fodale, V.; David, A.; Francesca, C. Lack of the Nlrp3 inflammasome improves mice recovery following traumatic brain injury. Front. Pharmacol. 2017, 8, 459. [CrossRef]

25. Campolo, M.; Ahmad, A.; Crupi, R.; Impellizzeri, D.; Morabito, R.; Esposito, E.; Cuzzocrea, S. Combination therapy with melatonin and dexamethasone in a mouse model of traumatic brain injury. J. Endocrinol. 2013, 217, 291-301. [CrossRef]

26. Zetterberg, H.; Blennow, K. Fluid biomarkers for mild traumatic brain injury and related conditions. Nat. Rev. Neurol. 2016, 12, 563. [CrossRef]

27. Wang, G.; Zhang, L.; Chen, X.; Xue, X.; Guo, Q.; Liu, M.; Zhao, J. Formylpeptide receptors promote the migration and differentiation of rat neural stem cells. Sci. Rep. 2016, 6, 25946. [CrossRef]

28. Braun, H.; Schäfer, K.; Höllt, V. $\beta$ III Tubulin-Expressing Neurons Reveal Enhanced Neurogenesis in Hippocampal and Cortical Structures after a Contusion Trauma in Rats. J. Neurotrauma 2002, 19, 975-983. [CrossRef]

29. Bye, N.; Carron, S.; Han, X.; Agyapomaa, D.; Ng, S.Y.; Yan, E.; Rosenfeld, J.V.; Morganti-Kossmann, M.C. Neurogenesis and glial proliferation are stimulated following diffuse traumatic brain injury in adult rats. J. Neurosci. Res. 2011, 89, 986-1000. [CrossRef]

30. Liberles, S.D.; Horowitz, L.F.; Kuang, D.; Contos, J.J.; Wilson, K.L.; Siltberg-Liberles, J.; Liberles, D.A.; Buck, L.B. Formyl peptide receptors are candidate chemosensory receptors in the vomeronasal organ. Proc. Natl. Acad. Sci. USA 2009, 106, 9842-9847. [CrossRef]

31. Cattaneo, F.; Guerra, G.; Ammendola, R. Expression and signaling of formyl-peptide receptors in the brain. Neurochem. Res. 2010, 35, 2018-2026. [CrossRef] [PubMed]

32. Gao, J.-L.; Schneider, E.H.; Dimitrov, E.L.; Haun, F.; Pham, T.M.; Mohammed, A.H.; Usdin, T.B.; Murphy, P.M. Reduced fear memory and anxiety-like behavior in mice lacking formylpeptide receptor 1 . Behav. Genet. 2011, 41, 724-733. [CrossRef] [PubMed]

33. Gao, J.L.; Lee, E.J.; Murphy, P.M. Impaired antibacterial host defense in mice lacking the N-formylpeptide receptor. J. Exp. Med. 1999, 189, 657-662. [CrossRef] [PubMed]

34. Cardini, S.; Dalli, J.; Fineschi, S.; Perretti, M.; Lungarella, G.; Lucattelli, M. Genetic ablation of the fpr1 gene confers protection from smoking-induced lung emphysema in mice. Am. J. Respir. Cell Mol. Biol. 2012, 47, 332-339. [CrossRef] [PubMed]

35. Peritore, A.F.; Crupi, R.; Scuto, M.; Gugliandolo, E.; Siracusa, R.; Impellizzeri, D.; Cordaro, M.; D'Amico, R.; Fusco, R.; Di Paola, R.; et al. The Role of Annexin A1 and Formyl Peptide Receptor 2/3 Signaling in Chronic Corticosterone-Induced Depression-Like behaviors and Impairment in Hippocampal-Dependent Memory. CNS Neurol. Disord. Drug Targets 2020, 19, 27-43. [CrossRef]

36. Gugliandolo, E.; D'Amico, R.; Cordaro, M.; Fusco, R.; Siracusa, R.; Crupi, R.; Impellizzeri, D.; Cuzzocrea, S.; Di Paola, R. Neuroprotective Effect of Artesunate in Experimental Model of Traumatic Brain Injury. Front. Neurol. 2018, 9, 590. [CrossRef]

37. Impellizzeri, D.; Cordaro, M.; Bruschetta, G.; Siracusa, R.; Crupi, R.; Esposito, E.; Cuzzocrea, S. N-Palmitoylethanolamine-Oxazoline as a new therapeutic strategy to control neuroinflammation: Neuroprotective effects in experimental models of spinal cord and brain injury. J. Neurotrauma 2017, 34, 2609-2623. [CrossRef]

38. Di Paola, R.; Fusco, R.; Gugliandolo, E.; Crupi, R.; Evangelista, M.; Granese, R.; Cuzzocrea, S. Co-micronized palmitoylethanolamide/polydatin treatment causes endometriotic lesion regression in a rodent model of surgically induced endometriosis. Front. Pharmacol. 2016, 7, 382. [CrossRef]

39. Siracusa, R.; Impellizzeri, D.; Cordaro, M.; Crupi, R.; Esposito, E.; Petrosino, S.; Cuzzocrea, S. Anti-Inflammatory and neuroprotective effects of Co-UltraPEALut in a mouse model of vascular dementia. Front. Neurol. 2017, 8, 233. [CrossRef]

40. Parrini, M.; Ghezzi, D.; Deidda, G.; Medrihan, L.; Castroflorio, E.; Alberti, M.; Baldelli, P.; Cancedda, L.; Contestabile, A. Aerobic exercise and a BDNF-mimetic therapy rescue learning and memory in a mouse model of Down syndrome. Sci. Rep. 2017, 7, 16825. [CrossRef] 
41. Cuadrado-Tejedor, M.; Hervias, I.; Ricobaraza, A.; Puerta, E.; Perez-Roldan, J.M.; Garcia-Barroso, C.; Franco, R.; Aguirre, N.; Garcia-Osta, A. Sildenafil restores cognitive function without affecting beta-amyloid burden in a mouse model of Alzheimer's disease. Br. J. Pharmacol. 2011, 164, 2029-2041. [CrossRef] [PubMed]

42. Cordaro, M.; Impellizzeri, D.; Siracusa, R.; Gugliandolo, E.; Fusco, R.; Inferrera, A.; Esposito, E.; Di Paola, R.; Cuzzocrea, S. Effects of a co-micronized composite containing palmitoylethanolamide and polydatin in an experimental model of benign prostatic hyperplasia. Toxicol. Appl. Pharmacol. 2017, 329, 231-240. [CrossRef] [PubMed]

43. Di Paola, R.; Impellizzeri, D.; Fusco, R.; Cordaro, M.; Siracusa, R.; Crupi, R.; Esposito, E.; Cuzzocrea, S. Ultramicronized palmitoylethanolamide (PEA-um ${ }^{\circledR}$ ) in the treatment of idiopathic pulmonary fibrosis. Pharmacol. Res. 2016, 111, 405-412. [CrossRef] [PubMed]

44. Kawai, T.; Akira, S. Signaling to NF-kappaB by Toll-like receptors. Trends Mol. Med. 2007, 13, 460-469. [CrossRef]

45. Clark, R.S.; Schiding, J.K.; Kaczorowski, S.L.; Marion, D.W.; Kochanek, P.M. Neutrophil accumulation after traumatic brain injury in rats: Comparison of weight drop and controlled cortical impact models. J. Neurotrauma 1994, 11, 499-506. [CrossRef]

46. Yen, T.L.; Chang, C.C.; Chung, C.L.; Ko, W.C.; Yang, C.H.; Hsieh, C.Y. Neuroprotective Effects of Platonin, a Therapeutic Immunomodulating Medicine, on Traumatic Brain Injury in Mice after Controlled Cortical Impact. Int. J. Mol. Sci. 2018, 19, 1100. [CrossRef]

47. Fusco, R.; Scuto, M.; Cordaro, M.; D’Amico, R.; Gugliandolo, E.; Siracusa, R.; Peritore, A.F.; Crupi, R.; Impellizzeri, D.; Cuzzocrea, S.; et al. N-Palmitoylethanolamide-Oxazoline Protects against Middle Cerebral Artery Occlusion Injury in Diabetic Rats by Regulating the SIRT1 Pathway. Int. J. Mol. Sci. 2019, $20,4845$. [CrossRef]

48. D’amico, R.; Fusco, R.; Gugliandolo, E.; Cordaro, M.; Siracusa, R.; Impellizzeri, D.; Peritore, A.F.; Crupi, R.; Cuzzocrea, S.; Di Paola, R. Effects of a new compound containing Palmitoylethanolamide and Baicalein in myocardial ischaemia/reperfusion injury in vivo. Phytomedicine 2019, 54, 27-42. [CrossRef]

49. Wang, X.; Seekaew, P.; Gao, X.; Chen, J. Traumatic Brain Injury Stimulates Neural Stem Cell Proliferation via Mammalian Target of Rapamycin Signaling Pathway Activation. eNeuro 2016, 3. [CrossRef]

50. Di Paola, R.; Fusco, R.; Gugliandolo, E.; D’Amico, R.; Cordaro, M.; Impellizzeri, D.; Perretti, M.; Cuzzocrea, S. Formyl peptide receptor 1 signalling promotes experimental colitis in mice. Pharmacol. Res. 2019, 141, 591-601. [CrossRef]

51. Viatour, P.; Merville, M.P.; Bours, V.; Chariot, A. Phosphorylation of NF-kappaB and IkappaB proteins: Implications in cancer and inflammation. Trends Biochem. Sci. 2005, 30, 43-52. [CrossRef] [PubMed]

52. Campolo, M.; Esposito, E.; Cuzzocrea, S. A controlled cortical impact preclinical model of traumatic brain injury. In Neurotrophic Factors; Springer: Berlin/Heidelberg, Germany, 2018; pp. 385-391.

53. Davalos, D.; Grutzendler, J.; Yang, G.; Kim, J.V.; Zuo, Y.; Jung, S.; Littman, D.R.; Dustin, M.L.; Gan, W.-B. ATP mediates rapid microglial response to local brain injury in vivo. Nat. Neurosci. 2005, 8, 752-758. [CrossRef] [PubMed]

54. Gehrmann, J.; Matsumoto, Y.; Kreutzberg, G.W. Microglia: Intrinsic immuneffector cell of the brain. Brain Res. Rev. 1995, 20, 269-287. [CrossRef]

55. Issekutz, A.C.; Issekutz, T.B. Cellular and Vascular Phenomena in Inflammation. Method Enzymol. 1988, 162, 301-320.

56. Kochanek, P.M.; Hallenbeck, J.M. Polymorphonuclear Leukocytes and Monocytes/Macrophages in the Pathogenesis of Cerebral-Ischemia and Stroke. Stroke 1992, 23, 1367-1379. [CrossRef] [PubMed]

57. Fusco, R.; D'amico, R.; Cordaro, M.; Gugliandolo, E.; Siracusa, R.; Peritore, A.F.; Crupi, R.; Impellizzeri, D.; Cuzzocrea, S.; Di Paola, R. Absence of formyl peptide receptor 1 causes endometriotic lesion regression in a mouse model of surgically-induced endometriosis. Oncotarget 2018, 9, 31355. [CrossRef]

58. Sengelov, H.; Boulay, F.; Kjeldsen, L.; Borregaard, N. Subcellular localization and translocation of the receptor for $\mathrm{N}$-formylmethionyl-leucyl-phenylalanine in human neutrophils. Biochem. J. 1994, 299, 473-479. [CrossRef]

59. Cowland, J.B.; Borregaard, N. The individual regulation of granule protein mRNA levels during neutrophil maturation explains the heterogeneity of neutrophil granules. J. Leukoc. Biol. 1999, 66, 989-995. [CrossRef] 
60. Hwang, T.-L.; Wang, C.-C.; Kuo, Y.-H.; Huang, H.-C.; Wu, Y.-C.; Kuo, L.-M.; Wu, Y.-H. The hederagenin saponin SMG-1 is a natural FMLP receptor inhibitor that suppresses human neutrophil activation. Biochem. Pharmacol. 2010, 80, 1190-1200. [CrossRef]

61. Dang, P.M.-C.; Stensballe, A.; Boussetta, T.; Raad, H.; Dewas, C.; Kroviarski, Y.; Hayem, G.; Jensen, O.N.; Gougerot-Pocidalo, M.-A.; El-Benna, J. A specific p47 phox-serine phosphorylated by convergent MAPKs mediates neutrophil NADPH oxidase priming at inflammatory sites. J. Clin. Investig. 2006, 116, 2033-2043. [CrossRef]

62. Xu, J.; Hsu, C.Y.; Liu, T.H.; Hogan, E.L.; Perot, P.L.; Tai, H.H. Leukotriene-B4 Release and Polymorphonuclear Cell Infiltration in Spinal-Cord Injury. J. Neurochem. 1990, 55, 907-912. [CrossRef] [PubMed]

63. Mori, T.; Wang, X.Y.; Jung, T.C.; Sumii, T.; Singhal, A.B.; Fini, M.E.; Dixon, C.E.; Alessandrini, A.; Lo, E.H. Mitogen-activated protein kinase inhibition in traumatic brain injury: In vitro and in vivo effects. J. Cereb. Blood Flow Metab. 2002, 22, 444-452. [CrossRef] [PubMed]

64. Pouliot, M.; Fiset, M.E.; Masse, M.; Naccache, P.H.; Borgeat, P. Adenosine up-regulates cyclooxygenase-2 in human granulocytes: Impact on the balance of eicosanoid generation. J. Immunol. 2002, 169, 5279-5286. [CrossRef] [PubMed]

65. Shojo, H.; Borlongan, C.V.; Mabuchi, T. Genetic and Histological Alterations Reveal Key Role of Prostaglandin Synthase and Cyclooxygenase 1 and 2 in Traumatic Brain Injury-Induced Neuroinflammation in the Cerebral Cortex of Rats Exposed to Moderate Fluid Percussion Injury. Cell Transplant. 2017, 26, 1301-1313. [CrossRef]

66. Mattson, M.; Meffert, M. Roles for NF-кB in nerve cell survival, plasticity, and disease. Cell Death Differ. 2006, 13, 852. [CrossRef]

67. Qian, H.; Li, Q.; Shi, W. Hyperbaric oxygen alleviates the activation of NLRP-3-inflammasomes in traumatic brain injury. Mol. Med. Rep. 2017, 16, 3922-3928. [CrossRef]

68. Liu, H.-D.; Li, W.; Chen, Z.-R.; Hu, Y.-C.; Zhang, D.-D.; Shen, W.; Zhou, M.-L.; Zhu, L.; Hang, C.-H. Expression of the NLRP3 inflammasome in cerebral cortex after traumatic brain injury in a rat model. Neurochem. Res. 2013, 38, 2072-2083. [CrossRef]

69. Ismael, S.; Nasoohi, S.; Ishrat, T. MCC950, the selective NLRP3 inflammasome inhibitor protects mice against traumatic brain injury. J. Neurotrauma 2018, 35, 1294-1303. [CrossRef]

70. Elliott, E.I.; Sutterwala, F.S. Initiation and perpetuation of NLRP 3 inflammasome activation and assembly. Immunol. Rev. 2015, 265, 35-52. [CrossRef]

71. Stover, J.F.; Schöning, B.; Beyer, T.F.; Woiciechowsky, C.; Unterberg, A.W. Temporal profile of cerebrospinal fluid glutamate, interleukin-6, and tumor necrosis factor- $\alpha$ in relation to brain edema and contusion following controlled cortical impact injury in rats. Neurosci. Lett. 2000, 288, 25-28. [CrossRef]

72. Rothwell, N. Interleukin-1 and neuronal injury: Mechanisms, modification, and therapeutic potential. Brain Behav. Immun. 2003, 17, 152-157. [CrossRef]

73. Bowie, A.; $\mathrm{O}^{\prime}$ Neill, L.A. Oxidative stress and nuclear factor- $\mathrm{\kappa} \mathrm{B}$ activation: A reassessment of the evidence in the light of recent discoveries. Biochem. Pharmacol. 2000, 59, 13-23. [CrossRef]

74. Orihara, Y.; Ikematsu, K.; Tsuda, R.; Nakasono, I. Induction of nitric oxide synthase by traumatic brain injury. Forensic Sci. Int. 2001, 123, 142-149. [CrossRef]

75. Gahm, C.; Holmin, S.; Wiklund, P.N.; Brundin, L.; Mathiesen, T. Neuroprotection by selective inhibition of inducible nitric oxide synthase after experimental brain contusion. J. Neurotrauma 2006, 23, 1343-1354. [CrossRef]

76. Morganti-Kossmann, M.C.; Satgunaseelan, L.; Bye, N.; Kossmann, T. Modulation of immune response by head injury. Injury 2007, 38, 1392-1400. [CrossRef]

77. Kernie, S.G.; Erwin, T.M.; Parada, L.F. Brain remodeling due to neuronal and astrocytic proliferation after controlled cortical injury in mice. J. Neurosci. Res. 2001, 66, 317-326. [CrossRef]

78. Zhao, M.; Liang, F.; Xu, H.; Yan, W.; Zhang, J. Methylene blue exerts a neuroprotective effect against traumatic brain injury by promoting autophagy and inhibiting microglial activation. Mol. Med. Rep. 2016, 13, 13-20. [CrossRef]

79. Loane, D.J.; Stoica, B.A.; Tchantchou, F.; Kumar, A.; Barrett, J.P.; Akintola, T.; Xue, F.; Conn, P.J.; Faden, A.I. Novel mGluR5 positive allosteric modulator improves functional recovery, attenuates neurodegeneration, and alters microglial polarization after experimental traumatic brain injury. Neurotherapeutics 2014, 11, 857-869. [CrossRef] 
80. Woodcock, T.; Morganti-Kossmann, M.C. The role of markers of inflammation in traumatic brain injury. Front. Neurol. 2013, 4, 18. [CrossRef]

81. Loane, D.J.; Kumar, A.; Stoica, B.A.; Cabatbat, R.; Faden, A.I. Progressive neurodegeneration after experimental brain trauma: Association with chronic microglial activation. J. Neuropathol. Exp. Neurol. 2014, 73, 14-29. [CrossRef]

82. Wang, X.; Gao, X.; Michalski, S.; Zhao, S.; Chen, J. Traumatic brain injury severity affects neurogenesis in adult mouse hippocampus. J. Neurotrauma 2016, 33, 721-733. [CrossRef] [PubMed]

83. Shin, M.K.; Jang, Y.H.; Yoo, H.J.; Kang, D.W.; Park, M.H.; Kim, M.K.; Song, J.H.; Kim, S.D.; Min, G.; You, H.K. $\mathrm{N}$-formyl-methionyl-leucyl-phenylalanine (fMLP) promotes osteoblast differentiation via the N-formyl peptide receptor 1-mediated signaling pathway in human mesenchymal stem cells from bone marrow. J. Biol. Chem. 2011, 286, 17133-17143. [CrossRef] [PubMed]

84. Viswanathan, A.; Painter, R.G.; Lanson, N.A.; Wang, G. Functional expression of N-formyl peptide receptors in human bone marrow-derived mesenchymal stem cells. Stem Cells 2007, 25, 1263-1269. [CrossRef] [PubMed]

85. Kim, M.-K.; Park, Y.J.; Kim, J.H.; Ryu, S.H.; Bae, Y.-S. Expression and functional role of formyl peptide receptor in human bone marrow-derived mesenchymal stem cells. FEBS Lett. 2007, 581, 1917-1922. [CrossRef]

86. Zhang, L.; Wang, G.; Chen, X.X.; Xue, X.; Guo, Q.N.; Liu, M.Y.; Zhao, J.H. Formyl peptide receptors promotes neural differentiation in mouse neural stem cells by ROS generation and regulation of PI3KAKT signaling. Sci. Rep. 2017, 7, 206. [CrossRef]

87. Le Belle, J.E.; Orozco, N.M.; Paucar, A.A.; Saxe, J.P.; Mottahedeh, J.; Pyle, A.D.; Wu, H.; Kornblum, H.I. Proliferative neural stem cells have high endogenous ROS levels that regulate self-renewal and neurogenesis in a PI3K/Akt-dependant manner. Cell Stem Cell 2011, 8, 59-71. [CrossRef]

(C) 2020 by the authors. Licensee MDPI, Basel, Switzerland. This article is an open access article distributed under the terms and conditions of the Creative Commons Attribution (CC BY) license (http://creativecommons.org/licenses/by/4.0/). 